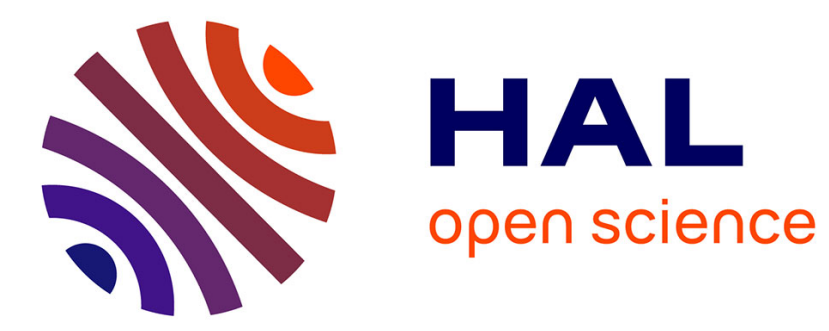

\title{
Exact converging bounds for Stochastic Dual Dynamic Programming via Fenchel duality
}

Vincent Leclère, Pierre Carpentier, Jean-Philippe Chancelier, Arnaud Lenoir, François Pacaud

\section{- To cite this version:}

Vincent Leclère, Pierre Carpentier, Jean-Philippe Chancelier, Arnaud Lenoir, François Pacaud. Exact converging bounds for Stochastic Dual Dynamic Programming via Fenchel duality. SIAM Journal on Optimization, 2020, 30 (2), pp.1223-1250. 10.1137/19M1258876 . hal-01744035v2

HAL Id: hal-01744035 https://hal-enpc.archives-ouvertes.fr/hal-01744035v2

Submitted on 18 Apr 2018

HAL is a multi-disciplinary open access archive for the deposit and dissemination of scientific research documents, whether they are published or not. The documents may come from teaching and research institutions in France or abroad, or from public or private research centers.
L'archive ouverte pluridisciplinaire HAL, est destinée au dépôt et à la diffusion de documents scientifiques de niveau recherche, publiés ou non, émanant des établissements d'enseignement et de recherche français ou étrangers, des laboratoires publics ou privés. 


\title{
Exact converging bounds for Stochastic Dual Dynamic Programming via Fenchel duality
}

\author{
Vincent Leclère, ${ }^{*}$ Pierre Carpentier ${ }^{\dagger}$ Jean-Philippe Chancelier, ${ }^{\ddagger}$ \\ Arnaud Lenoir ${ }^{\S}$ and François Pacaud ${ }^{\Uparrow}$
}

April 12, 2018

\begin{abstract}
The Stochastic Dual Dynamic Programming (SDDP) algorithm has become one of the main tools to address convex multistage stochastic optimal control problem. Recently a large amount of work has been devoted to improve the convergence speed of the algorithm through cut-selection and regularization, or to extend the field of applications to non-linear, integer or risk-averse problems. However one of the main downside of the algorithm remains the difficulty to give an upper bound of the optimal value, usually estimated through Monte Carlo methods and therefore difficult to use in the algorithm stopping criterion.

In this paper we present a dual SDDP algorithm that yields a converging exact upper bound for the optimal value of the optimization problem. Incidently we show how to compute an alternative control policy based on an inner approximation of Bellman value functions instead of the outer approximation given by the standard SDDP algorithm. We illustrate the approach on an energy production problem involving zones of production and transportation links between the zones. The numerical experiments we carry out on this example show the effectiveness of the method.
\end{abstract}

\section{Introduction}

In this paper, we consider a risk neutral multistage stochastic optimization problem, with continuous decision variables. We adopt the stochastic optimal control point of view, that is, we work with explicit control and state variables in order to deal with an explicit dynamics of the system and to obtain an interpretation of the multipliers associated to the dynamics.

\subsection{Stochastic optimization problem in discrete time}

Let $(\Omega, \mathcal{A}, \mathbb{P})$ be a probability space, where $\Omega$ is the set of possible outcomes, $\mathcal{A}$ the associated $\sigma$-field and $\mathbb{P}$ the probability measure. We denote by $\llbracket 0, T \rrbracket$ the discrete time span $\{0,1, \ldots, T\}$, and we define upon it three processes $\boldsymbol{X}=\left\{\boldsymbol{X}_{t}\right\}_{t \in \llbracket 0, T \rrbracket}, \boldsymbol{U}=\left\{\boldsymbol{U}_{t}\right\}_{t \in \llbracket 1, T-1 \rrbracket}$ and $\boldsymbol{\xi}=\left\{\boldsymbol{\xi}_{t}\right\}_{t \in \llbracket 1, T \rrbracket}$ where for all $t, \boldsymbol{X}_{t}: \Omega \rightarrow \mathbb{R}^{n_{x}}, \boldsymbol{U}_{t}: \Omega \rightarrow \mathbb{R}^{n_{u}}$ and $\boldsymbol{\xi}_{t}: \Omega \rightarrow \mathbb{R}^{n_{\xi}}$ are random variables representing

\footnotetext{
*Université Paris-Est, CERMICS (ENPC) - vincent.leclere@enpc.fr

†UMA, ENSTA ParisTech, Université Paris-Saclay - pierre.carpentier@ensta-paristech.fr

‡Université Paris-Est, CERMICS (ENPC) - chancelier@cermics.enpc.fr

$\S \mathrm{EDF}$ - arnaud.lenoir@edf.fr

ฯ CERMICS (ENPC) - Efficacity - francois.pacaud@enpc.com
} 
respectively the state, the control and the noise variables. The state process $\boldsymbol{X}$ is assumed to follow the linear dynamics

$$
\begin{aligned}
& \boldsymbol{X}_{0}=x_{0}, \\
& \boldsymbol{X}_{t+1}=A_{t} \boldsymbol{X}_{t}+B_{t+1} \boldsymbol{U}_{t+1}+C_{t+1} \boldsymbol{\xi}_{t+1} \quad \forall t \in \llbracket 0, T-1 \rrbracket,
\end{aligned}
$$

where $x_{0}$ is the given initial state at time 0 and where $A_{t} \in \mathbb{R}^{n_{x} \times n_{x}}, B_{t+1} \in \mathbb{R}^{n_{x} \times n_{u}}$ and $C_{t+1} \in \mathbb{R}^{n_{x} \times n_{\xi}}$ are given deterministic matrices. We moreover assume that both the control and the state variables are subject to bound constraints, that is, for all $t \in \llbracket 0, T-1 \rrbracket, \underline{u}_{t+1} \leq \boldsymbol{U}_{t+1} \leq$ $\bar{u}_{t+1}$, and $\underline{x}_{t+1} \leq \boldsymbol{X}_{t+1} \leq \bar{x}_{t+1}$ and satisfy a linear coupling constraint

$$
D_{t} \boldsymbol{X}_{t}+E_{t+1} \boldsymbol{U}_{t+1}+G_{t+1} \boldsymbol{\xi}_{t+1} \leq 0 \quad \forall t \in \llbracket 0, T-1 \rrbracket .
$$

where $D_{t} \in \mathbb{R}^{n_{c} \times n_{x}}, E_{t+1} \in \mathbb{R}^{n_{c} \times n_{u}}$ and $G_{t+1} \in \mathbb{R}^{n_{c} \times n_{\xi}}$ are given matrices. In particular, state and control variables take values in compact subsets of $\mathbb{R}^{n_{x}}$ and $\mathbb{R}^{n_{u}}$ respectively.

We assume that the problem has a Hazard-Decision ${ }^{1}$ information structure, that is, the decision at time $t$ is taken knowing the noise that affects the system between $t$ and $t+1$. Accordingly, the decision $\boldsymbol{U}_{t+1}$ is a function of the uncertainties up to time $t+1$, which means that $\boldsymbol{U}_{t+1}$ has to be measurable with respect to the $\sigma$-field $\mathcal{F}_{t+1}$ generated by the uncertainties $\left(\boldsymbol{\xi}_{1}, \cdots, \boldsymbol{\xi}_{t+1}\right)$. We write this non anticipativity constraint as, $\boldsymbol{U}_{t+1} \preceq \mathcal{F}_{t+1}$, for all $t \in \llbracket 0, T-1 \rrbracket$.

Finally, the cost incurred at each time $t \in \llbracket 0, T-1 \rrbracket$ is a linear function $a_{t}^{\top} \boldsymbol{X}_{t}+b_{t+1}^{\top} \boldsymbol{U}_{t+1}$ with $a_{t} \in \mathbb{R}^{n_{x}}$ and $b_{t+1} \in \mathbb{R}^{n_{u}}$, and the cost incurred at the final time $T$ is $K\left(\boldsymbol{X}_{T}\right)$ where $K$ is a polyhedral, hence convex lower semi-continuous, function. The results obtained in this paper for a polyhedral function can be easily adapt to the case where $K$ is a convex lower semi-continuous function, Lipschitz-continuous on its domain.

Gathering all these elements, we get the following stochastic optimization problem:

$$
\begin{array}{lll}
\min _{\boldsymbol{X}, \boldsymbol{U}} \mathbb{E}\left[\sum_{t=0}^{T-1}\left(a_{t}^{\top} \boldsymbol{X}_{t}+b_{t+1}^{\top} \boldsymbol{U}_{t+1}\right)+K\left(\boldsymbol{X}_{T}\right)\right], & \\
\text { s.t. } & \boldsymbol{X}_{0}=x_{0}, & \\
& \boldsymbol{X}_{t+1}=A_{t} \boldsymbol{X}_{t}+B_{t+1} \boldsymbol{U}_{t+1}+C_{t+1} \boldsymbol{\xi}_{t+1} & \\
& \underline{u}_{t+1} \leq \boldsymbol{U}_{t+1} \leq \bar{u}_{t+1} & \forall t \in \llbracket 0, T-1 \rrbracket, \\
& \underline{x}_{t+1} \leq \boldsymbol{X}_{t+1} \leq \bar{x}_{t+1} & \forall t \in \llbracket 0, T-1 \rrbracket, \\
& D_{t} \boldsymbol{X}_{t}+E_{t+1} \boldsymbol{U}_{t+1}+G_{t+1} \boldsymbol{\xi}_{t+1} \leq 0 & \forall t \in \llbracket 0, T-1 \rrbracket, \\
& \boldsymbol{U}_{t+1} \preceq \mathcal{F}_{t+1} & \forall t \in \llbracket 0, T-1 \rrbracket .
\end{array}
$$

We make the following assumption throughout the paper.

Assumption 1 (discrete white noise). The noise sequence $\left\{\boldsymbol{\xi}_{t}\right\}_{t \in \llbracket 1, T \rrbracket}$ is assumed to be a sequence of independent variables with finite support.

As it is well known, independence is of paramount importance to obtain Dynamic Programming equation, while finiteness of the support is required both to be able to compute exactly the expectation and for theoretical convergence reasons.

\footnotetext{
${ }^{1}$ Wait-and-see in the Stochastic Programming terminology
} 


\subsection{Stochastic Dual Dynamic Programming and its weaknesses}

Thanks to white noise Assumption 1, we can solve Problem (1) by the Dynamic Programming approach (see the two reference books Bellman [1957] and Bertsekas 2005 for further details). This approach leads to the so-called Bellman's value functions $V_{t}$, such that $V_{t}(x)$ is the optimal value of the problem when starting at time $t$ with state $\boldsymbol{X}_{t}=x$. These functions are obtained by solving the following recursive Bellman equation:

$$
\begin{aligned}
& V_{T}(x)=K(x), \\
& V_{t}(x)=\mathbb{E}\left[\inf _{u_{t+1}, x_{t+1}} a_{t}^{\top} x+b_{t+1}^{\top} u_{t+1}+V_{t+1}\left(x_{t+1}\right)\right] \\
& \text { s.t. } \quad x_{t+1}=A_{t} x+B_{t+1} u_{t+1}+C_{t+1} \xi_{t+1}, \\
& \quad \underline{u}_{t+1} \leq u_{t+1} \leq \bar{u}_{t+1}, \\
& \quad \underline{x}_{t+1} \leq x_{t+1} \leq \bar{x}_{t+1}, \\
& D_{t} x+E_{t+1} u_{t+1}+G_{t+1} \xi_{t+1} \leq 0 .
\end{aligned}
$$

When the state variable takes a finite number of possible values, we can solve the Bellman equation by exhaustive exploration of the state, yielding the exact solution of the problem. In the continuous linear-convex case, we can rely on polyhedral approximations. The Stochastic Dual Dynamic Programming algorithm (SDDP) builds polyhedral approximations $\underline{V}_{t}$ of the functions $V_{t}$ by using a sampled nested Benders decomposition (see Philpott and Guan 2008, Girardeau et al. 2014], Guigues [2016] and Guigues [2017] for the convergence of this approach). The polyhedral value functions $\underline{V}_{t}$ computed by SDDP are outer approximations of the functions $V_{t}$ at each stage, that is, $\underline{V}_{t} \leq V_{t}$, so that the value $\underline{v}_{0}=\underline{V}_{0}\left(x_{0}\right)$ is an exact (deterministic) lower bound on the optimal value $v_{0}=V_{0}\left(x_{0}\right)$ of Problem (1).

Functions $\underline{V}_{t}$ can also be used to derive an admissible strategy, whose associated expected cost $\bar{v}_{0}$ gives an upper bound of the optimization problem value. Unfortunately, computing the expectation is usually out of reach, and we need to rely on some approximate computation. The most common way to perform that task is based on the Monte Carlo approach: it consists in simulating the control strategy induced by functions $\underline{V}_{t}$ along a (large) number $M$ of noise scenarios, and then computing the arithmetic mean $\widehat{v}_{0}^{M}$ of the scenarios cost and the associated empirical standard deviation $\widehat{\sigma}_{0}^{M}$. The value $\widehat{v}_{0}^{M}$ is an approximate (statistical) upper bound of the optimal value of Problem (1). Moreover, it is easy to obtain an asymptotic $\alpha$-confidence interval $\left[\widehat{v}_{0}^{M}-z_{\alpha} \widehat{\sigma}_{0}^{M}, \widehat{v}_{0}^{M}+z_{\alpha} \widehat{\sigma}_{0}^{M}\right]$. Here $1-\alpha \in[0,1]$ is a chosen confidence level and $z_{\alpha}=$ $\Phi^{-1}(1-\alpha), \Phi$ being the cumulative distribution function of the standard normal distribution.

The classical way to use this statistical upper bound in SDDP, as presented in Pereira and Pinto 1991], consists in testing at each iteration of the algorithm if the available exact lower bound $\underline{v}_{0}$ is greater than the $\alpha$-confidence lower bound $\widehat{v}_{0}^{M}-z_{\alpha} \widehat{\sigma}_{0}^{M}$, and to stop the algorithm in that case. Such a stopping criterion raises at least two difficulties: the Monte Carlo simulation increases the computational burden of SDDP, and the stopping test does not give any guarantee of convergence of the algorithm.

The first difficulty can be tackled by parallelizing the $M$ simulations involved in the evaluation of the upper bound, and also by calculating the empirical mean $\widehat{v}_{0}$ over the last $\bar{k}$ iterations of the algorithm, thus enlarging the sample size from $M$ to $\bar{k} M$ without additional computation (see [Shapiro et al., 2012, §3.2]). The second difficulty induced by this stopping criterion has been analyzed in Shapiro [2011]: the larger the standard deviation $\widehat{\sigma}_{0}$ and the confidence $(1-\alpha)$ are, the sooner the algorithm will be stopped. The author proposes another criterion based on the difference between the $\alpha$-confidence upper bound $\widehat{v}_{0}+z_{\alpha} \widehat{\sigma}_{0}$ and the exact lower bound $\underline{v}_{0}$ up to a prescribed accuracy level $\varepsilon$. Note that if $\varepsilon<z_{\alpha} \widehat{\sigma}_{0}$, this stopping test is not necessarily 
convergent, in the sense that the stopping criterion might not be met in a finite time. An interesting view on the class of stopping criteria in terms of statistical hypothesis tests has been given in Homem-de Mello et al. [2011]: the authors compare different hypothesis tests of optimality ${ }^{2}$ and so they find the stopping criteria proposed by Pereira and Pinto 1991, Shapiro 2011, as well as another one which ensures an upper bound on the probability of incorrectly claiming convergence (type II error). Moreover, the simulation scenarios are obtained using Quasi-Monte Carlo or Latin Hypercube Sampling rather than raw Monte carlo, so that the accuracy of the upper bound is increased. Nevertheless, all these stopping criteria are based on a statistical evaluation and thus do not guarantee that the gap is almost surely smaller than some $\varepsilon$.

A different approach consists in building polyhedral inner approximations $\bar{V}_{t}$ of the Bellman functions $V_{t}$ at each stage, that is, $\bar{V}_{t} \geq V_{t}$. A deterministic upper bound $\bar{V}_{0}\left(x_{0}\right)$ of the optimal value of Problem (1) thus becomes available, and it is then possible to perform a stopping test of SDDP on the almost-sure gap $\bar{V}_{0}\left(x_{0}\right)-\underline{V}_{0}\left(x_{0}\right)$. Such a test, giving a guarantee on the algorithm convergence has been investigated in Philpott et al. 2013. More precisely, starting from a polyhedral inner approximation $\bar{V}_{t+1}$ at time $t+1$, and choosing an arbitrary sequence of points $x_{t}^{1}, \ldots, x_{t}^{J_{t}}$, the authors show how to compute a value $q_{t}^{j}$ at each point $x_{t}^{j}$ such that $q_{t}^{j} \geq V_{t}\left(x_{t}^{j}\right)$. The inner polyhedral approximation $\bar{V}_{t}$ is then obtained from the pairs $\left\{\left(x_{t}^{j}, q_{t}^{j}\right)\right\}_{j \in \llbracket 1, J_{t} \rrbracket}$. A delicate issue when devising the inner approximations is the choice of the points $x_{t}^{j}$ defining the polyhedral functions $\bar{V}_{t}$. The authors suggest to use points generated by some other algorithm, such as SDDP. Another approach involving inner and outer approximations of the Bellman functions is described in Baucke et al. [2017], whose main feature is that the algorithm presented herein is fully deterministic.

\subsection{Contents of the paper}

In Sect. 2, we introduce the formalism of linear Bellman operators for a large class of optimization problem, we define its dual linear Bellman operator and we enlighten the relationships between them thanks to the Fenchel conjugate. We also present the SDDP algorithm that applies to a sequence of function recursively defined through linear Bellman operators. We apply in Sect. 3 the conjugacy results obtained in Sect. 2 to obtain a recursion on the dual value functions, on which we can apply the abstract SDDP algorithm, yielding a dual SDDP algorithm for solving Problem (1). The main result of this section is that we eventually obtain an exact upper bound over the value of Problem (1). In Sect. 4, we show how to build inner approximations of Bellman functions associated to Problem (1) thanks to outer approximations computed by the dual SDDP algorithm. This inner-approximation induces a control strategy converging toward an optimal one (see Theorem 29. Furthermore, the expected cost incurred by this strategy is shown to be lower than the exact upper bound obtained in Sect. 3. Ultimately, in Sect. 5, we illustrate all the presented methodology on an energy management problem inspired by Électricité de France, at the European scale. The results show, on the one hand that having at disposal an exact upper bound in SDDP allows to devise a more efficient stopping test for SDDP than the usual ones based on a Monte Carlo approach, and on the other hand that the strategy based on the inner approximation of the Bellman functions outperforms the usual strategy obtained using standard outer approximations.

\subsection{Notations}

- $\llbracket i, j \rrbracket$ denotes the set of integer between $i$ and $j$.

\footnotetext{
${ }^{2}$ such as: $\left(\mathrm{H}_{0}: \bar{v}_{0}=\underline{v}_{0}\right)$ against $\left(\mathrm{H}_{1}: \bar{v}_{0} \neq \underline{v}_{0}\right)$
} 
- $\Omega$ denotes a finite set of cardinality $|\Omega|$ supporting a probability distribution $\mathbb{P}$ : $\Omega=\left\{\omega_{1}, \ldots, \omega_{|\Omega|}\right\}$ and $\mathbb{P}=\left(\pi_{1}, \ldots, \pi_{|\Omega|}\right)$.

- Random variables are denoted using bold uppercase letters (such as $\boldsymbol{Z}: \Omega \rightarrow \mathbb{Z}$ ), and their realizations are denoted using lowercase letters $(z \in \mathbb{Z})$.

- $\boldsymbol{X}: \Omega \rightarrow \mathbb{X}$ corresponds to the state, $\boldsymbol{U}: \Omega \rightarrow \mathbb{U}$ to the control, $\boldsymbol{\xi}: \Omega \rightarrow \Xi$ to the noise.

- $V_{t}: \mathbb{X}_{t} \rightarrow \mathbb{R}$ is the Bellman value function associated to Problem (1) at time $t$.

- $\mathcal{D}_{t}=\left[V_{t}\right]^{\star}$ is the dual value function associated to Problem (1) at time $t$.

- $\mathcal{B}$ is the Bellman operator associated to a generic linear problem, with associated solution operator $\mathcal{S}$, and dual operator denoted $\mathcal{B}^{\ddagger}$.

- $\mathcal{T}$ is the Bellman operator associated to Problem (1), its dual being denoted $\mathcal{T}^{\ddagger}$.

- Underlined notation (e.g. $\underline{V}$ ) corresponds to a lower approximation of a function (e.g $V$ ). Overlined notation (e.g. $\bar{V}$ ) denotes an upper approximation.

\section{Linear Bellman operators}

This self-contained section is devoted to the definition and properties of linear Bellman operators (LBOs). In 2.1 we present the abstract formalism of LBOs that allows to write Dynamic Programming equations in a compact manner. In $\$ 2.2$ we show that the Fenchel transform of a LBO is also a LBO. In 2.3 we present an abstract version of the SDDP algorithm adapted to the LBO formulation.

\subsection{Linear Bellman operator}

We first introduce the notion of linear Bellman operator, which is a particular class of Bellman operators (see Bertsekas 2005) associated to stochastic optimal control problems where costs and constraints are linear.

We consider an abstract probability space $(\Omega, \mathcal{A}, \mathbb{P})$. Recall that $\Omega$ is a finite set (see Assumption (1) and assume that the $\sigma$-field $\mathcal{A}$ is generated by all the singletons of $\Omega$. We denote by $\mathcal{L}^{0}\left(\mathbb{R}^{n_{x}} ; \overline{\mathbb{R}}\right)$ the set of functions defined on $\mathbb{R}^{n_{x}}$ and taking values in $[-\infty,+\infty]$, and by $\mathcal{L}^{0}\left(\Omega, \mathcal{A} ; \mathbb{R}^{n_{x}}\right)$ the space of $\mathbb{R}^{n_{x}}$-valued measurable functions defined on $(\Omega, \mathcal{A}, \mathbb{P})$.

Remark 1. Since we suppose that the set $\Omega$ is finite, every function defined on $\Omega$ is measurable and a property that holds almost surely is a property that holds for every $\omega \in \Omega$. In particular $\mathcal{L}^{0}\left(\Omega, \mathcal{A} ; \mathbb{R}^{n}\right)$ is a finite-dimensional space whathever $n \in \mathbb{N}$.

Definition 2. An operator $\mathcal{B}: \mathcal{L}^{0}\left(\mathbb{R}^{n_{x}} ; \overline{\mathbb{R}}\right) \rightarrow \mathcal{L}^{0}\left(\mathbb{R}^{n_{x}} ; \overline{\mathbb{R}}\right)$ is said to be a linear Bellman operator (LBO) if it is defined as follows

$$
\begin{aligned}
\mathcal{B}: \mathcal{L}^{0}\left(\mathbb{R}^{n_{x}} ; \overline{\mathbb{R}}\right) & \rightarrow \mathcal{L}^{0}\left(\mathbb{R}^{n_{x}} ; \overline{\mathbb{R}}\right) \\
R & \mapsto \mathcal{B}(R): x \mapsto \inf _{(\boldsymbol{U}, \boldsymbol{Y}) \in \mathcal{L}^{0}\left(\Omega, \mathcal{A} ; \mathbb{R}^{n_{x}}\right) \times \mathcal{L}^{0}\left(\Omega, \mathcal{A} ; \mathbb{R}^{\left.n_{u}\right)}\right.} \\
\text { s.t. } & \operatorname{Tx}+\mathcal{W}_{u}(\boldsymbol{U})+\boldsymbol{W}_{y}(\boldsymbol{Y}) \leq \boldsymbol{H},
\end{aligned}
$$

where $\mathcal{W}_{u}: \mathcal{L}^{0}\left(\Omega, \mathcal{A} ; \mathbb{R}^{n_{u}}\right) \rightarrow \mathcal{L}^{0}\left(\Omega, \mathcal{A} ; \mathbb{R}^{n_{c}}\right)$ and $\mathcal{W}_{y}: \mathcal{L}^{0}\left(\Omega, \mathcal{A} ; \mathbb{R}^{n_{x}}\right) \rightarrow \mathcal{L}^{0}\left(\Omega, \mathcal{A} ; \mathbb{R}^{n_{c}}\right)$ are two linear operators. Here, $\boldsymbol{U}$ and $\boldsymbol{Y}$ are two decision random variables respectively defined on $\mathbb{R}^{n_{u}}$ 
and $\mathbb{R}^{n_{x}}$. The two random variables $\boldsymbol{C}: \Omega \rightarrow \mathbb{R}^{n_{u}}$ and $\boldsymbol{H}: \Omega \rightarrow \mathbb{R}^{n_{c}}$ are exogenous uncertainties in Problem (2) and we note $\boldsymbol{\xi}=(\boldsymbol{C}, \boldsymbol{H})$. Deterministic matrix $T \in \mathbb{R}^{n_{c} \times n_{x}}$ is given data.

$W e$ denote by $\mathcal{S}(R)$ the set valued mapping giving, for a given $x \in \mathbb{R}^{n_{x}}$, the set of optimal solutions $\boldsymbol{Y}$ of Problem (2):

$$
\begin{aligned}
& \mathcal{S}(R): \mathbb{R}^{n_{x}} \rightarrow \mathcal{L}^{0}\left(\Omega, \mathcal{A} ; \mathbb{R}^{n_{x}}\right) \\
& x \mapsto \underset{\boldsymbol{Y} \in \mathcal{L}^{0}\left(\Omega, \mathcal{A} ; \mathbb{R}^{n_{x}}\right)}{\arg \min }\left(\inf _{\boldsymbol{U} \in \mathcal{L}^{0}\left(\Omega, \mathcal{A} ; \mathbb{R}^{n_{u}}\right)} \mathbb{E}\left[\boldsymbol{C}^{\top} \boldsymbol{U}+R(\boldsymbol{Y})\right]\right), \\
& \text { s.t. } \quad T x+\mathcal{W}_{u}(\boldsymbol{U})+\mathcal{W}_{y}(\boldsymbol{Y}) \leq \boldsymbol{H} .
\end{aligned}
$$

Let $\mathcal{G}: \mathbb{R}^{n_{x}} \rightrightarrows \mathcal{L}^{0}\left(\Omega, \mathcal{A} ; \mathbb{R}^{n_{u}}\right) \times \mathcal{L}^{0}\left(\Omega, \mathcal{A} ; \mathbb{R}^{n_{x}}\right)$ be the set valued mapping defined by

$$
\mathcal{G}(x):=\left\{(\boldsymbol{U}, \boldsymbol{Y}) \mid T x+\mathcal{W}_{u}(\boldsymbol{U})+\mathcal{W}_{y}(\boldsymbol{Y}) \leq \boldsymbol{H}\right\}
$$

With domain $\operatorname{dom}(\mathcal{G})=\left\{x \in \mathbb{R}^{n_{x}} \mid \mathcal{G}(x) \neq \emptyset\right\}$. Further, we say that $\mathcal{B}$ is compact if $\mathcal{G}$ is compact-valued with non-empty compact domain.

Note that, using the definition of the set valued mapping $\mathcal{G}$, we have that

$$
\mathcal{B}(R)(x)=\inf _{\left.(\boldsymbol{U}, \boldsymbol{Y}) \in \mathcal{L}^{0}\left(\Omega, \mathcal{A} ; \mathbb{R}^{n_{x}}\right) \times \mathbb{R}^{n_{u}}\right)} \mathbb{E}\left[\boldsymbol{C}^{\top} \boldsymbol{U}+\mathbb{I}_{\mathcal{G}(x)}(\boldsymbol{U}, \boldsymbol{Y})\right]+\mathbb{E}[R(\boldsymbol{Y})],
$$

where $\mathbb{I}_{A}$ is the characteristic function of a set $A$ :

$$
\mathbb{I}_{A}(x)=\left\{\begin{array}{cl}
0 & \text { if } x \in A \\
+\infty & \text { otherwise }
\end{array}\right.
$$

Example 3. We give some classical examples of operators $\mathcal{W}_{u}$ and $\mathcal{W}_{y}$ involved in Definition 2 of $\mathcal{B}$. We stress out that $\mathcal{W}$ is a linear operator over a space of random variables, and we describe the associated adjoint operator, that is, the linear operator $\mathcal{W}^{\dagger}$ such that $\langle\boldsymbol{X}, \mathcal{W}(\boldsymbol{Y})\rangle=$ $\left\langle\mathcal{W}^{\dagger}(\boldsymbol{X}), \boldsymbol{Y}\right\rangle$, with $\langle\boldsymbol{X}, \boldsymbol{Y}\rangle=\mathbb{E}\left[\boldsymbol{X}^{\top} \boldsymbol{Y}\right]$.

- Linear point-wise operator:

$$
\begin{aligned}
& \mathcal{W}: \mathcal{L}^{0}\left(\Omega, \mathcal{A} ; \mathbb{R}^{n_{x}}\right) \rightarrow \mathcal{L}^{0}\left(\Omega, \mathcal{A} ; \mathbb{R}^{n_{c}}\right) \\
& (\omega \mapsto \boldsymbol{Y}(\omega)) \mapsto(\omega \mapsto A \boldsymbol{Y}(\omega)) .
\end{aligned}
$$

Such an operator allows to encode an almost sure constraint, and $\mathcal{W}^{\dagger}(\boldsymbol{X})=A^{\top} \boldsymbol{X}$.

- Linear expected operator:

$$
\begin{array}{rlll}
\mathcal{W}: & \mathcal{L}^{0}\left(\Omega, \mathcal{A} ; \mathbb{R}^{n_{x}}\right) & \rightarrow & \mathcal{L}^{0}\left(\Omega, \mathcal{A} ; \mathbb{R}^{n_{c}}\right) \\
(\omega \mapsto \boldsymbol{Y}(\omega)) & \mapsto \quad(\omega \mapsto A \mathbb{E}(\boldsymbol{Y}))
\end{array}
$$

Such an operator allows to encode a constraint in expectation, and $\mathcal{W}^{\dagger}(\boldsymbol{X})=A^{\top} \mathbb{E}(\boldsymbol{X})$.

- Linear conditional operator: given a sub $\sigma$-field $\mathcal{F}$ of $\mathcal{A}$,

$$
\begin{aligned}
& \mathcal{W}: \mathcal{L}^{0}\left(\Omega, \mathcal{A} ; \mathbb{R}^{n_{x}}\right) \quad \rightarrow \quad \mathcal{L}^{0}\left(\Omega, \mathcal{A} ; \mathbb{R}^{n_{c}}\right) \\
& (\omega \mapsto \boldsymbol{Y}(\omega)) \mapsto(\omega \mapsto A \mathbb{E}[\boldsymbol{Y} \mid \mathcal{F}](\omega)),
\end{aligned}
$$

Such an operator allows to encode, for example, measurability constraints and $\mathcal{W}^{\dagger}(\boldsymbol{X})=$ $A^{\top} \mathbb{E}[\boldsymbol{X} \mid \mathcal{F}]$. 
Of course, any linear combination of these three kinds of operator is also linear. 1976 .

We define the key notion of relatively complete recourse, introduced in Rockafellar and Wets

Definition 4. Let $Q \in \mathcal{L}^{0}\left(\mathbb{R}^{n_{x}} ; \overline{\mathbb{R}}\right)$ and $\mathcal{B}$ a $L B O$. We say that the pair $(\mathcal{B}, Q)$ satisfies a relatively complete recourse $(R C R)$ assumption if $\operatorname{dom}(\mathcal{B}(Q))=\operatorname{dom}(\mathcal{G})$, that is, if

$$
\forall x \in \operatorname{dom}(\mathcal{G}), \exists(\boldsymbol{U}, \boldsymbol{Y}) \in \mathcal{G}(x) \text { such that } \mathbb{P}(\boldsymbol{Y} \in \operatorname{dom}(Q))=1 .
$$

Remark 5. Note that if $\mathcal{B}$ is compact and $(\mathcal{B}, Q)$ satisfy the $\mathrm{RCR}$ assumption, then $\mathcal{B}(Q)$ is finite at some point $x_{0}$.

We now turn to properties of LBOs and polyhedral functions.

Proposition 6. Let $R$ be a function of $\mathcal{L}^{0}\left(\mathbb{R}^{n_{x}} ; \overline{\mathbb{R}}\right)$ and let $\mathcal{B}$ be a LBO. Then we have the following properties.

1. If $R$ is convex, then $\mathcal{B}(R)$ is convex.

2. If $R$ is polyhedral, then $\mathcal{B}(R)$ is polyhedral.

3. If $R \geq \tilde{R}$, then $\mathcal{B}(R) \geq \mathcal{B}(\tilde{R})$.

Proof. The probability set $\Omega$ being finite, we denote by $u$ (resp. $y, c, h$ ) the vectors concatenating all possible values of $\boldsymbol{U}(\operatorname{resp} . \boldsymbol{Y}, \boldsymbol{C}, \boldsymbol{H})$ over the set $\Omega$, that is $u=\left(u_{1}, \ldots, u_{|\Omega|}\right)$. Then the extensive formulation of Constraint $2 \mathrm{~b}$ is

$$
\widetilde{T} x+\widetilde{W}_{u} u+\widetilde{W}_{y} y \leq h,
$$

where $\widetilde{T}, \widetilde{W}_{u}$ and $\widetilde{W}_{y}$ are adequate matrices deduced from $T, \mathcal{W}_{u}$ and $\mathcal{W}_{y}$. Problem 2 rewrites

$$
\mathcal{B}(R)(x)=\inf _{u, y} J(R)(x, u, y)
$$

with

$$
J(R)(x, u, y)=\sum_{\omega=1}^{|\Omega|} \pi_{\omega}\left(c_{\omega}^{\top} u_{\omega}+R\left(y_{\omega}\right)\right)+\mathbb{I}_{\left\{\widetilde{T} x+\widetilde{W}_{u} u+\widetilde{W}_{y} y \leq h\right\}}(x, u, y)
$$

1. If $R$ is convex, then $J(R)$ is jointly convex in $(x, u, y)$ so that $\mathcal{B}(R)$ is a convex function.

2. If $R$ is polyhedral, then $J(R)$ is polyhedral in $(x, u, y)$, and thus $\mathcal{B}(R)$ is a polyhedral function (see Borwein and Lewis, 2010, Prop 5.1.8]).

3. From $R \geq \tilde{R}$, we deduce that $J(R) \geq J(\tilde{R})$, and thus $\mathcal{B}(R) \geq \mathcal{B}(\tilde{R})$.

The proof is complete.

Remark 7. Assume that function $R$ is proper and polyhedral. Then, under relatively complete recourse (see Definition 4), and if $\mathcal{B}(R)$ is finite at some point, $\mathcal{B}(R)$ is a proper polyhedral function. Furthermore, if $\mathcal{B}(R)(x)$ is finite, solving its (linear programming) dual generates a supporting hyperplane at point $x$ of the function $\mathcal{B}(R)$, that is, a pair $(\lambda, \beta) \in \mathbb{R}^{n_{x}} \times \mathbb{R}$ such that

$$
\left\{\begin{array}{l}
\langle\lambda, \cdot\rangle+\beta \leq \mathcal{B}(R)(\cdot) \\
\langle\lambda, x\rangle+\beta=\mathcal{B}(R)(x) .
\end{array}\right.
$$

Such hyperplanes, or cuts, are of paramount importance for the SDDP algorithm. 
The following proposition establish a link between the Lipschitz constants of $R$ and $\mathcal{B}(R)$. Je rajouterais bien l'hypothese de compacite sur $\mathcal{B}$ pour pas avoir d'ennuis avec les $-\infty$

Proposition 8. Let $R$ be a proper polyhedral function of $\mathcal{L}^{0}\left(\mathbb{R}^{n_{x}} ; \overline{\mathbb{R}}\right)$ and let $\mathcal{B}$ be a LBO. Assume that $(\mathcal{B}, R)$ satisfies the $R C R$ assumption and that $\mathcal{B}(R)$ is finite at some point. If $R$ is Lipschitz (for the $L_{1}$-norm) with constant $L_{R}$, then $\mathcal{B}(R)$ is also Lipschitz on its domain (which is $\operatorname{dom}(\mathcal{G})$ ) with constant $\phi\left(L_{R}\right)=\left(\|\boldsymbol{C}\|_{\infty}+L_{R}\right) \kappa_{W}\|T\|_{\infty}$, where $\kappa_{W}$ is a constant associated to the linear operator $\left(\mathcal{W}_{u}, \mathcal{W}_{y}\right)$.

Proof. Consider $x_{1}$ (resp. $x_{2}$ ) an element in $\operatorname{dom}\left(\mathcal{B}(R)\right.$ ), and denote by $Z_{1}$ (resp. $Z_{2}$ ) the polyhedron of optimal solutions of Problem (2). These polyhedrons are non-empty thanks to the RCR assumption. Let $\left(\boldsymbol{U}_{1}, \boldsymbol{Y}_{1}\right) \in Z_{1}$ be fixed, from [Shapiro et al., 2009, Theorem 7.12], there exists a positive constant $\kappa_{W}$ such that

$$
\inf _{\left(\boldsymbol{U}_{2}, \boldsymbol{Y}_{2}\right) \in Z_{2}}\left\|\left(\boldsymbol{U}_{1}, \boldsymbol{Y}_{1}\right)-\left(\boldsymbol{U}_{2}, \boldsymbol{Y}_{2}\right)\right\|_{1} \leq \kappa_{W}\left\|T\left(x_{1}-x_{2}\right)\right\|_{1} \leq \kappa_{W}\|T\|_{\infty}\left\|x_{1}-x_{2}\right\|_{1} .
$$

Let $\left(\boldsymbol{U}_{2}^{\sharp}, \boldsymbol{Y}_{2}^{\sharp}\right) \in Z_{2}$ be an optimal solution of the above minimization problem. Then we have that

$$
\begin{aligned}
\mathcal{B}(R)\left(x_{1}\right) & =\mathbb{E}\left[\boldsymbol{C}^{\top} \boldsymbol{U}_{1}+R\left(\boldsymbol{Y}_{1}\right)\right] \\
& \left.\leq \mathbb{E}\left[\boldsymbol{C}^{\top} \boldsymbol{U}_{2}^{\sharp}+\boldsymbol{C}^{\top}\left(\boldsymbol{U}_{1}-\boldsymbol{U}_{2}^{\sharp}\right)+R\left(\boldsymbol{Y}_{2}^{\sharp}\right)+L_{R}\left\|\boldsymbol{Y}_{2}^{\sharp}-\boldsymbol{Y}_{1}\right\|_{1}\right)\right] \\
& \leq \mathcal{B}(R)\left(x_{2}\right)+\|\boldsymbol{C}\|_{\infty}\left\|\boldsymbol{U}_{1}-\boldsymbol{U}_{2}^{\sharp}\right\|_{1}+L_{R}\left\|\boldsymbol{Y}_{1}-\boldsymbol{Y}_{2}^{\sharp}\right\|_{1} \\
& \leq \mathcal{B}(R)\left(x_{2}\right)+\left(\|\boldsymbol{C}\|_{\infty}+L_{R}\right) \kappa_{W}\|T\|_{\infty}\left\|x_{1}-x_{2}\right\|_{1} .
\end{aligned}
$$

Exchanging $x_{1}$ and $x_{2}$ in the previous majoration leads to the reverse inequality, combining both leads to the desired Lipschitz property.

\subsection{Fenchel transform of a LBO}

We now define the dual linear Bellman operator $\mathcal{B}^{\ddagger}$ of a linear Bellman operator $\mathcal{B}$.

Definition 9. Let $\mathcal{B}$ be a LBO (see Definition (2)). We denote by $\mathcal{B}^{\ddagger}$ the dual LBO of $\mathcal{B}$, defined, for a given function $Q \in \mathcal{L}^{0}\left(\mathbb{R}^{n_{x}} ; \overline{\mathbb{R}}\right)$ and for any $\lambda \in \mathbb{R}^{n_{x}}$, by

$$
\begin{aligned}
\mathcal{B}^{\ddagger}(Q)(\lambda)=\inf _{\boldsymbol{\mu} \in \mathcal{L}^{0}\left(\Omega, \mathcal{A} ; \mathbb{R}^{n_{x}}\right), \boldsymbol{\nu} \in \mathcal{L}^{0}\left(\Omega, \mathcal{A} ; \mathbb{R}^{n_{c}}\right)} & \mathbb{E}\left[-\boldsymbol{\mu}^{\top} \boldsymbol{H}+Q(\boldsymbol{\nu})\right] \\
\text { s.t. } \quad & T^{\top} \mathbb{E}[\boldsymbol{\mu}]+\lambda=0 \\
& \mathcal{W}_{u}^{\dagger}(\boldsymbol{\mu})=\boldsymbol{C} \\
& \mathcal{W}_{y}^{\dagger}(\boldsymbol{\mu})=\boldsymbol{\nu} \\
& \boldsymbol{\mu} \leq 0,
\end{aligned}
$$

where $\mathcal{W}_{u}^{\dagger}$ (resp. $\mathcal{W}_{y}^{\dagger}$ ) is the adjoint operator of $\mathcal{W}_{u}$ (resp. $\left.\mathcal{W}_{y}\right)$. We define the dual constraint set valued mapping

$$
\mathcal{G}^{\ddagger}(\lambda)=\left\{(\boldsymbol{\mu}, \boldsymbol{\nu}) \in \mathbb{R}^{n_{x}+n_{c}} \mid T^{\top} \mathbb{E}[\boldsymbol{\mu}]+\lambda=0, \mathcal{W}_{u}^{\dagger}(\boldsymbol{\mu})=\boldsymbol{C}, \mathcal{W}_{y}^{\dagger}(\boldsymbol{\mu})=\boldsymbol{\nu}, \boldsymbol{\mu} \leq 0\right\} .
$$

Note that straightforward computations show that $\left(\mathcal{B}^{\ddagger}\right)^{\ddagger}=\mathcal{B}$.

The next proposition exhibit the relationship between $\mathcal{B}^{\ddagger}$ and the Fenchel transform of $\mathcal{B}$. 
Theorem 10. Let $R$ be a proper polyhedral function, $\mathcal{B}$ be a compact LBO (see Definition 2), such that the pair $(\mathcal{B}, R)$ satisfies the $R C R$ assumption. Then $\mathcal{B}(R)$ is a proper polyhedral function and we have

$$
[\mathcal{B}(R)]^{\star}=\mathcal{B}^{\ddagger}\left([R]^{\star}\right)
$$

Proof. First note that as $\mathcal{B}$ is compact, $\mathcal{G}$ has non-empty compact domain, and thus $\mathcal{B}(R)$ is finite at some point by the RCR assumption.

During the proof we denote $\langle\boldsymbol{X}, \boldsymbol{Y}\rangle=\mathbb{E}\left(\boldsymbol{X}^{\top} \boldsymbol{Y}\right), \mathcal{R}(\boldsymbol{Y})=\mathbb{E}(R(\boldsymbol{Y}))$, and

$$
\begin{aligned}
\mathcal{K}(x, \boldsymbol{Y})=\min _{\boldsymbol{U}} & \langle\boldsymbol{C}, \boldsymbol{U}\rangle \\
\text { s.t. } & T x+\mathcal{W}_{u}(\boldsymbol{U})+\mathcal{W}_{y}(\boldsymbol{Y}) \leq \boldsymbol{H} .
\end{aligned}
$$

By definition, we have

$$
\mathcal{B}(R)(x)=\inf _{\boldsymbol{Y}} \mathcal{K}(x, \boldsymbol{Y})+\mathcal{R}(\boldsymbol{Y})
$$

Thus, for any $x^{\star} \in \mathbb{R}^{n_{x}}$, we have

$$
\begin{aligned}
{[\mathcal{B}(R)]^{\star}\left(x^{\star}\right) } & =\sup _{x \in \mathbb{R}^{n_{x}}}\left\{x^{\top} x^{\star}-\inf _{\boldsymbol{Y}}\{\mathcal{K}(x, \boldsymbol{Y})+\mathcal{R}(\boldsymbol{Y})\}\right\} \\
& =-\inf _{\boldsymbol{Y}}\{\mathcal{R}(\boldsymbol{Y})-\underbrace{\sup _{x \in \mathbb{R}^{n_{x}}} x^{\top} x^{\star}-\mathcal{K}(x, Y)}_{:=\Phi(\boldsymbol{Y})}\}
\end{aligned}
$$

As $R$ is polyhedral proper, so is $\mathcal{R}$. By construction $\mathcal{K}$ is polyhedral. By compacity of $\mathcal{B}$, the minimization in $\boldsymbol{U}$ is over a compact, thus $\mathcal{K}>-\infty$. Further, as $\operatorname{dom}(\mathcal{B}(R))=\operatorname{dom}(\mathcal{G}) \neq \emptyset, \mathcal{K}$ is proper. Note that for $x \notin \operatorname{dom}(\mathcal{G})$ we have $\mathcal{K}(x, \boldsymbol{Y})=+\infty$, thus $\Phi(\boldsymbol{Y})=\sup _{x \in \operatorname{dom}(\mathcal{G})} x^{\top} x^{\star}-$ $\mathcal{K}(x, Y)$. Consequently, $-\Phi$ is polyhedral proper as $\operatorname{dom}(\mathcal{G})$ is a non empty compact set. Finally, the RCR assumption ensures that $\operatorname{dom}(-\Phi) \cap \operatorname{dom}(\mathcal{R}) \neq \emptyset$. Now, using Fenchel-Duality (see Proposition 32 we have that

$$
[\mathcal{B}(R)]^{\star}\left(x^{\star}\right)=-\sup _{\boldsymbol{Y}^{\star}} \Phi_{\star}\left(\boldsymbol{Y}^{\star}\right)-\mathcal{R}^{\star}\left(\boldsymbol{Y}^{\star}\right)=\inf _{\boldsymbol{Y}^{\star}} \mathcal{R}^{\star}\left(\boldsymbol{Y}^{\star}\right)-\Phi_{\star}\left(\boldsymbol{Y}^{\star}\right)
$$

where $\mathcal{R}^{\star}\left(\boldsymbol{Y}^{\star}\right)=\mathbb{E}\left(R^{\star}\left(\boldsymbol{Y}^{\star}\right)\right)$ and

$$
\begin{aligned}
\Phi_{\star}\left(\boldsymbol{Y}^{\star}\right)= & \inf _{\boldsymbol{Y}}\left\langle\boldsymbol{Y}^{\star}, \boldsymbol{Y}\right\rangle-\Phi(\boldsymbol{Y}) \\
= & \inf _{\boldsymbol{Y}}\left\langle\boldsymbol{Y}^{\star}, \boldsymbol{Y}\right\rangle-\sup _{x}\left\{x^{\top} x^{\star}-\mathcal{K}(x, \boldsymbol{Y})\right\} \\
= & \inf _{x, \boldsymbol{Y}}\left\langle\boldsymbol{Y}^{\star}, \boldsymbol{Y}\right\rangle-x^{\top} x^{\star}+\mathcal{K}(x, \boldsymbol{Y}) \\
= & \inf _{x, \boldsymbol{Y}, \boldsymbol{U}}\left\langle\boldsymbol{Y}^{\star}, \boldsymbol{Y}\right\rangle-x^{\top} x^{\star}+\langle\boldsymbol{C}, \boldsymbol{U}\rangle \\
& \text { s.t. } T x+\mathcal{W}_{u}(\boldsymbol{U})+\mathcal{W}_{y}(\boldsymbol{Y}) \leq \boldsymbol{H}
\end{aligned}
$$

As $\operatorname{dom}(\mathcal{G}) \neq \emptyset$, there exists a primal feasible solution to the above linear program, and by 
duality we have

$$
\begin{aligned}
\Phi_{\star}\left(\boldsymbol{Y}^{\star}\right)= & \sup _{\boldsymbol{\lambda} \geq 0}-\langle\boldsymbol{\lambda}, \boldsymbol{H}\rangle+\inf _{x}\left\{-x^{\top} x^{\star}+\left\langle T^{\top} \boldsymbol{\lambda}, x\right\rangle\right\}+\inf _{\boldsymbol{Y}}\left\{\left\langle\boldsymbol{Y}^{\star}, \boldsymbol{Y}\right\rangle+\left\langle\mathcal{W}_{y}^{\dagger}(\boldsymbol{\lambda}), \boldsymbol{Y}\right\rangle\right\} \\
& +\inf _{\boldsymbol{U}}\left\{\langle\boldsymbol{C}, \boldsymbol{U}\rangle+\left\langle\mathcal{W}_{u}^{\dagger}(\boldsymbol{\lambda}), \boldsymbol{U}\right\rangle\right\} \\
= & \sup _{\boldsymbol{\lambda} \geq 0}-\langle\boldsymbol{\lambda}, \boldsymbol{H}\rangle \\
\text { s.t. } & \mathcal{W}_{y}^{\dagger}(\boldsymbol{\lambda})=-\boldsymbol{Y}^{\star} \\
& \mathcal{W}_{u}^{\dagger}(\boldsymbol{\lambda})=-\boldsymbol{C} \\
& T^{\top} \mathbb{E}(\boldsymbol{\lambda})=x^{\star}
\end{aligned}
$$

Finally,

$$
\begin{array}{rl}
{[\mathcal{B}(R)]^{\star}\left(x^{\star}\right)=\inf _{\boldsymbol{Y}^{\star}, \boldsymbol{\lambda} \geq 0}} & \mathbb{E}\left(\boldsymbol{H}^{\top} \boldsymbol{\lambda}+R^{\star}\left(\boldsymbol{Y}^{\star}\right)\right) \\
\text { s.t. } & T^{\top} \mathbb{E}(\boldsymbol{\lambda})=x^{\star} \\
& \mathcal{W}_{y}^{\dagger}(\boldsymbol{\lambda})=-\boldsymbol{Y}^{\star} \\
& \mathcal{W}_{u}^{\dagger}(\boldsymbol{\lambda})=-\boldsymbol{C},
\end{array}
$$

which is equivalent to (7) with the correspondence $x^{\star} \rightarrow \lambda, \boldsymbol{\lambda} \rightarrow \boldsymbol{\mu}$ and $\boldsymbol{Y}^{\star} \rightarrow \boldsymbol{\nu}$.

\subsection{An abstract SDDP algorithm}

We now consider a sequence of functions $\left\{R_{t}\right\}_{t \in \llbracket 0, T \rrbracket}$ that follows the Bellman recursion

$$
\left\{\begin{array}{l}
R_{T}=K \\
R_{t}=\mathcal{B}_{t}\left(R_{t+1}\right) \quad \forall t \in \llbracket 0, T-1 \rrbracket,
\end{array}\right.
$$

where $K$ is a proper polyhedral function, and a sequence of LBOs $\left\{\mathcal{B}_{t}\right\}_{t \in \llbracket 0, T-1 \rrbracket}$ given by

$$
\begin{array}{rl}
\mathcal{B}_{t}(R)(x)=\inf _{\boldsymbol{U}, \boldsymbol{Y}} & \mathbb{E}\left[\boldsymbol{C}_{t}^{\top} \boldsymbol{U}+R(\boldsymbol{Y})\right] \\
\text { s.t. } & T_{t} x+\mathcal{W}_{t}^{u}(\boldsymbol{U})+\mathcal{W}_{t}^{y}(\boldsymbol{Y}) \leq \boldsymbol{H}_{t},
\end{array}
$$

with associated set valued mappings $\left\{\mathcal{G}_{t}\right\}_{t \in \llbracket 0, T-1 \rrbracket}$ :

$$
\mathcal{G}_{t}(x):=\left\{(\boldsymbol{U}, \boldsymbol{Y}) \mid T_{t} x+\mathcal{W}_{t}^{u}(\boldsymbol{U})+\mathcal{W}_{t}^{y}(\boldsymbol{Y}) \leq \boldsymbol{H}_{t}\right\},
$$

and associated solution operators $\left\{\mathcal{S}_{t}\right\}_{t \in \llbracket 0, T-1 \rrbracket}$ :

$$
\begin{array}{rl}
\mathcal{S}_{t}(R)(x)=\underset{\boldsymbol{Y}}{\arg \min } \inf _{\boldsymbol{U}} & \mathbb{E}\left[\boldsymbol{C}_{t}^{\top} \boldsymbol{U}+R(\boldsymbol{Y})\right] \\
\text { s.t. } & T_{t} x+\mathcal{W}_{t}^{u}(\boldsymbol{U})+\mathcal{W}_{t}^{y}(\boldsymbol{Y}) \leq \boldsymbol{H}_{t} .
\end{array}
$$

We now give an extension of the RCR Definition 4 
Definition 11. Let $\left\{\mathcal{B}_{t}\right\}_{t \in \llbracket 0, T-1 \rrbracket}$ be a sequence of LBOs, with $\operatorname{dom}\left(\mathcal{G}_{t}\right) \neq \emptyset$, and $\left\{R_{t}\right\}_{t \in \llbracket 0, T \rrbracket}$ be defined by the Bellman recursion $\left[10\right.$. We say that the sequence $\left\{\mathcal{B}_{t}\right\}_{t \in \llbracket 0, T-1 \rrbracket}$ is $K$-compatible if,

$$
\forall t \in \llbracket 0, T-1 \rrbracket, \quad \forall x \in \operatorname{dom}\left(\mathcal{G}_{t}\right), \quad \forall\left(\boldsymbol{U}_{t+1}, \boldsymbol{Y}_{t+1}\right) \in \mathcal{G}_{t}(x), \quad \mathbb{P}\left(\boldsymbol{Y}_{t+1} \in \operatorname{dom}\left(\mathcal{G}_{t+1}\right)\right)=1,
$$

where by convention $\operatorname{dom}\left(\mathcal{G}_{T}\right)=\operatorname{dom}(K)$.

Remark 12. Note that the natural extension of the relatively complete assumption (see Definition (4) would be asking that, for all $t \in \llbracket 0, T-1 \rrbracket$, the pair $\left(\mathcal{B}_{t}, R_{t+1}\right)$ satisfies the RCR assumption, that is,

$$
\forall t \in \llbracket 0, T-1 \rrbracket, \quad \forall x \in \operatorname{dom}\left(\mathcal{G}_{t}\right), \quad \exists\left(\boldsymbol{U}_{t+1}, \boldsymbol{Y}_{t+1}\right) \in \mathcal{G}_{t}(x), \quad \mathbb{P}\left(\boldsymbol{Y}_{t+1} \in \operatorname{dom}\left(\mathcal{G}_{t+1}\right)\right)=1 .
$$

At first glance, assuming compatibility of LBOs seems to be stronger than this assumption. Indeed, we require that every admissible control leads to an admissible future state, instead of only assuming existence of one such control.

In fact the constraint $\boldsymbol{Y}_{t+1} \in \operatorname{dom}\left(\mathcal{G}_{t+1}\right) \mathbb{P}$-a.s. is implicit in the definition of $\mathcal{B}_{t}\left(R_{t+1}\right)$, since a control $\left(\boldsymbol{U}_{t+1}, \boldsymbol{Y}_{t+1}\right)$ such that $\boldsymbol{Y}_{t+1} \notin \operatorname{dom}\left(\mathcal{G}_{t+1}\right)=\operatorname{dom}\left(R_{t+1}\right)$ leads to an infinite value of $\mathcal{B}_{t}\left(R_{t+1}\right)(x)$. More precisely, consider a sequence of LBOs $\left\{\mathcal{B}_{t}^{e}\right\}_{t \in \llbracket 0, T-1 \rrbracket}$, with associated operators $\left\{\mathcal{G}_{t}^{e}\right\}_{t \in \llbracket 0, T \rrbracket}$ satisfying $[12]$, and define

$$
\left\{\begin{array}{l}
R_{T}=K \\
R_{t}=\mathcal{B}_{t}^{e}\left(R_{t+1}\right) \quad \forall t \in \llbracket 0, T-1 \rrbracket .
\end{array}\right.
$$

Then, we can define a new sequence of LBOs $\left\{\mathcal{B}_{t}\right\}_{t \in \llbracket 0, T-1 \rrbracket}$, where $\mathcal{B}_{t}$ is equal to $\mathcal{B}_{t}^{e}$ with the additional constraint that $\boldsymbol{Y}_{t+1} \in \operatorname{dom}\left(\mathcal{G}_{t+1}^{e}\right) \mathbb{P}$-a.s.. In this case we can easily see that the sequence $\left\{\mathcal{B}_{t}\right\}_{t \in \llbracket 0, T-1 \rrbracket}$ is $K$-compatible, and that $\left\{R_{t}\right\}_{t \in \llbracket 0, T \rrbracket}$ follows the Bellman recursion 10$\rangle$.

Finally, note that constructing $\mathcal{B}_{t}$ from $\mathcal{B}_{t}^{e}$ does not require multistage constraint propagation. Without loss of generality we will assume compatibility instead of $(12)$, this is useful to ensure that all state generated in the forward pass of the SDDP algorithm are admissible states.

SDDP is an algorithm that iteratively constructs finite lower polyhedral approximations of the sequence of functions $\left\{R_{t}\right\}_{t \in \llbracket 0, T-1 \rrbracket}$ given by Equation (10). Starting from an initial point $x_{0} \in \mathbb{R}^{n_{x}}$, the algorithm determines in a forward pass a sequence of states $\left(x_{t}^{k}\right)_{t \in \llbracket 0, T \rrbracket}$ at which the approximations of the sequence $\left\{R_{t}\right\}_{t \in \llbracket 0, T-1 \rrbracket}$ will be refined in the backward pass. More precisely, a pseudo-code describing the abstract SDDP algorithm is given in Algorithm 1.

Lemma 13. Assume that $R_{0}\left(x_{0}\right)$ is finite and that $\left(\mathcal{B}_{t}\right)_{t \in \llbracket 0, T-1 \rrbracket}$ is a $K$-compatible sequence of LBO. Then, the (abstract) SDDP Algorithm 1 is well defined and there exists a sequence

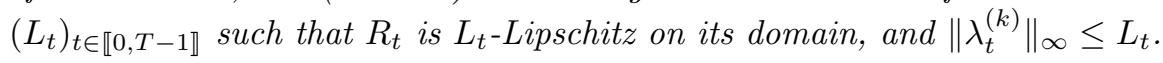

Proof. We prove by induction that $x_{t}^{(k)}$ is well defined during the forward passes of SDDP. Let $t=0$. By assumption, $x_{0}^{k} \in \operatorname{dom}\left(\mathcal{G}_{0}\right)$. So $x_{1}^{k}=\boldsymbol{X}_{1}^{(k)}\left(\omega^{k}\right)$ exists as solution of a finite valued LP. Let $t \geq 1$. By induction hypothesis, we suppose that $x_{t}^{k}$ is well defined and belongs to $\operatorname{dom}\left(\mathcal{G}_{t}\right)$. We set $x_{t+1}^{k}=\boldsymbol{X}_{t+1}^{(k)}\left(\omega^{k}\right)$, which is well defined as solution of a finite value LP. By assumption the sequence $\left\{\mathcal{B}_{t}\right\}_{t \in \llbracket 0, T-1 \rrbracket}$ is $K$-compatible, hence $x_{t+1}^{k} \in \operatorname{dom}\left(\mathcal{G}_{t+1}\right)$, thus proving the result at time $t+1$. 
Data: Initial point $x_{0}$

Set $\underline{R}_{t}^{(0)} \equiv-\infty$

for $k=0,1, \ldots$ do

// Forward Pass : compute a set of trial points $\left\{x_{t}^{k}\right\}_{t \in \llbracket 0, T \rrbracket}$

Randomly select $\omega^{k} \in \Omega$;

Set $x_{0}^{k}=x_{0}$;

for $t: 0 \rightarrow T-1$ do

$$
\text { select } \boldsymbol{X}_{t+1}^{k} \in \mathcal{S}_{t}\left(\underline{R}_{t+1}^{k}\right)\left(x_{t}^{k}\right) ; \quad \text { // see Definition 2 }
$$

end

// Backward Pass : refine the lower-approximations at the trial points

Set $\underline{R}_{T}^{k+1}=K$;

for $t: T-1 \rightarrow 0$ do

$\theta_{t}^{k+1}=\mathcal{B}_{t}\left(\underline{R}_{t+1}^{k+1}\right)\left(x_{t}^{k}\right)$

// cut coefficients (see Remark 7)

$\lambda_{t}^{k+1} \in \partial \mathcal{B}_{t}\left(\underline{R}_{t+1}^{k+1}\right)\left(x_{t}^{k}\right) ;$

$\beta_{t}^{k+1}:=\theta_{t}^{k+1}-\left\langle\lambda_{t}^{k+1}, \bar{x}_{t}^{k}\right\rangle$;

set $\mathcal{C}_{t}^{k+1}: x \mapsto\left\langle\lambda_{t}^{k+1}, x\right\rangle+\beta_{t}^{k+1}$

// new cut

$\underline{R}_{t}^{k+1}:=\max \left\{\underline{R}_{t}^{k}, \mathcal{C}_{t}^{k+1}\right\}$;

end

If some stopping test is satisfied STOP ;

end

Algorithm 1: Abstract SDDP algorithm

We now prove by backward induction that $\lambda_{t}^{(k)}$ is well defined during the backward passes of SDDP, and that there exists $L_{t}$ such that $\left\|\lambda_{t}^{(k)}\right\|_{\infty} \leq L_{t}$. As $K$ is given and $L_{T}$ Lipschitzcontinuous, the property is direct for $t=T$. Let $t \leq T-1$. Assume that induction hypothesis holds for $t+1$. Then, by Proposition 8 , we know that $\mathcal{B}_{t}\left(\underline{R}_{t+1}^{k+1}\right)$ is $L_{t}$-Lipschitz. We set $\lambda_{t}^{k+1} \in$ $\partial \mathcal{B}_{t}\left(\underline{R}_{t+1}^{k+1}\right)\left(x_{t}^{k}\right)$, which is well defined as subgradient of a finite-valued polyhedral function. As $\mathcal{B}_{t}\left(\underline{R}_{t+1}^{k+1}\right)$ is $L_{t}$-Lipschitz on its domain, we are able to choose $\lambda_{t}^{k+1}$ such that $\left\|\lambda_{t}^{(k+1)}\right\|_{\infty} \leq L_{t}$.

From Lemma 13 we have the boundness of $\lambda_{t}^{(k)}$, from which we can easily adapt the proof of Girardeau et al. 2014.

Proposition 14. Assume that $R_{0}\left(x_{0}\right)$ is finite and that $\left(\mathcal{B}_{t}\right)_{t \in \llbracket 0, T-1 \rrbracket}$ is a $K$-compatible sequence of LBO. Further assume that, for all $t \in \llbracket 0, T \rrbracket$ there exists compact sets $X_{t}$ such that, for all $k$, $x_{t}^{k} \in X_{t}$. In particular this is the case if $\mathcal{B}_{t}$ is compact for all $t$.

Then, the abstract SDDP algorithm generates a non-decreasing sequence $\left(\underline{R}_{t}^{(k)}\right)_{k \in \mathbb{N}}$ of lower approximation of $R_{t}$, and $\lim _{k} \underline{R}_{0}^{(k)}\left(x_{0}\right)=R_{0}\left(x_{0}\right)$.

This algorithm is abstract in the sense that it only requires a sequence of LBOs. In the following section we show how it can be applied to approximate the Bellman value functions $\left\{V_{t}\right\}_{t \in \llbracket 0, T \rrbracket}$, or to approximate the Fenchel transform of these functions. 


\section{Primal and dual SDDP}

In this section we recall the usual SDDP algorithm applied to Problem (1). Next, leveraging the results of Sect. 2, we introduce a dual SDDP algorithm, which is the abstract SDDP algorithm applied to the dual value functions. This eventually gives an exact upper bound over the value of Problem (1). In this section, we denote by $V_{t}$ the primal value functions, and by $\mathcal{D}_{t}=\left[V_{t}\right]^{\star}$ the dual value functions.

\subsection{Primal SDDP}

\subsubsection{Primal Dynamic Programming equations}

We consider Problem (1) under the By the discrete white noise Assumption 1, we can solve Problem (1) through Dynamic Programming, computing backward the value functions $\left\{V_{t}\right\}_{t \in \llbracket 0, T \rrbracket}$ given by

$$
\left\{\begin{array}{l}
V_{T}=K+\mathbb{I}_{\underline{x}_{T} \leq \cdot \leq \bar{x}_{T}}, \\
V_{t}=\mathcal{T}_{t}^{e}\left(V_{t+1}\right),
\end{array}\right.
$$

where the primal Bellman operator $\mathcal{T}_{t}^{e}: \mathcal{L}^{0}\left(\mathbb{R}^{n_{x}} ; \overline{\mathbb{R}}\right) \rightarrow \mathcal{L}^{0}\left(\mathbb{R}^{n_{x}} ; \overline{\mathbb{R}}\right)$ is defined as follows:

$$
\begin{array}{rl}
\mathcal{T}_{t}^{e}(R): x \mapsto \inf _{\boldsymbol{U}_{t+1}, \boldsymbol{X}_{t+1}} & \mathbb{E}\left[a_{t}^{\top} x+b_{t+1}^{\top} \boldsymbol{U}_{t+1}+R\left(\boldsymbol{X}_{t+1}\right)\right] \\
\text { s.t. } & \boldsymbol{X}_{t+1}=A_{t} x+B_{t+1} \boldsymbol{U}_{t+1}+C_{t+1} \boldsymbol{\xi}_{t+1}, \\
& D_{t} x+E_{t+1} \boldsymbol{U}_{t+1}+G_{t+1} \boldsymbol{\xi}_{t+1} \leq 0 \\
& \underline{u}_{t+1} \leq \boldsymbol{U}_{t+1} \leq \bar{u}_{t+1} \\
& \underline{x}_{t} \leq x \leq \bar{x}_{t} .
\end{array}
$$

Constraint (14e) ensures that if $x$ does not satisfies $\underline{x}_{t} \leq x \leq \bar{x}_{t}$, then $\mathcal{T}_{t}{ }^{e}(R)(x)=\infty$. By Definition 2 , the operator $\mathcal{T}_{t}^{e}$ is a LBO. The associated set valued mapping $\mathcal{G}_{t}^{e}$ is defined by

$$
\mathcal{G}_{t}^{e}(x):=\left\{\left(\boldsymbol{U}_{t+1}, \boldsymbol{X}_{t+1}\right), \text { s.t. constraints } 14 \mathrm{~b}-14 \mathrm{e} \text { are satisfied }\right\} .
$$

We make the following assumptions.

\section{Assumption 2.}

1. The function $K$ is polyhedral.

2. For all $t \in \llbracket 0, T-1 \rrbracket$, and all $x \in \operatorname{dom}\left(\mathcal{G}_{t}^{e}\right)$, there exists $\left(\boldsymbol{U}_{t+1}, \boldsymbol{X}_{t+1}\right) \in \mathcal{G}_{t}^{e}(x)$, such that $\boldsymbol{X}_{t+1} \in \operatorname{dom}\left(\mathcal{G}_{t+1}^{e}\right)$, where by convention $\operatorname{dom}\left(\mathcal{G}_{T}^{e}\right)=\operatorname{dom}(K)$.

3. Problem (1) is finite valued.

Remark 15. Point 2 of Assumption 2, is equivalent to asking that for all $t \in \llbracket 0, T-1 \rrbracket$, the pair $\left(\mathcal{T}_{t}^{e}, V_{t+1}\right)$ follows the (RCR) assumption as defined in Definition 4. Note that these assumption can be checked $t$ by $t$, that is, without requiring backward constraint propagation.

In order to produce admissible state in the forward pass of SDDP, we follow Remark 12, and define a more constrained primal LBO $\mathcal{T}_{t}$ by adding to Problem $(14)$ the following constraint

$$
\boldsymbol{X}_{t+1} \in \operatorname{dom}\left(\mathcal{G}_{t+1}^{e}\right) \text {. }
$$


The associated set valued mapping $\mathcal{G}_{t}$ is thus defined by

$$
\mathcal{G}_{t}(x):=\left\{\left(\boldsymbol{U}_{t+1}, \boldsymbol{X}_{t+1}\right) \text {, s.t. constraints 14b } 14 \mathrm{f} \text { are satisfied }\right\},
$$

and is compact valued with compact domain. Further, we have the Bellman recursion

$$
\left\{\begin{array}{l}
V_{T}=K \\
V_{t}=\mathcal{T}_{t}\left(V_{t+1}\right)
\end{array}\right.
$$

For the sake of notational simplicity, we assume from now on that constraints $114 \mathrm{~d})-(14 \mathrm{e})$ 14f are embedded in Constraint 14c) (see an example of such an embedding in Appendix A.4.

Lemma 16. Under Assumption 2. $\left\{\mathcal{T}_{t}\right\}_{t \in \llbracket 0, T-1 \rrbracket}$ is a compatible sequence of compact LBOs. Further, for any $t \in \llbracket 0, T-1 \rrbracket$, we have

$$
\mathcal{T}_{t}(R): x \mapsto \mathbb{E}\left(\widehat{\mathcal{T}}_{t}(R)\left(x, \boldsymbol{\xi}_{t+1}\right)\right),
$$

where

$$
\begin{aligned}
\widehat{\mathcal{T}}_{t}(R):(x, \xi) \mapsto \inf _{u_{t+1}, x_{t+1}} & a_{t}^{\top} x+b_{t+1}^{\top} u_{t+1}+R\left(x_{t+1}\right), \\
\text { s.t. } & x_{t+1}=A_{t} x+B_{t+1} u_{t+1}+C_{t+1} \xi, \\
& D_{t} x+E_{t+1} u_{t+1}+G_{t+1} \xi \leq 0 .
\end{aligned}
$$

Proof. As Problem (1) is finite valued, the domain of each set valued mapping $\mathcal{G}_{t}$ is non empty. Further, as $\mathcal{G}_{t}$ is compact valued with compact domain, each LBO $\mathcal{B}_{t}$ is compact (see Definition 2).

Then, the reformulation as Equations $(17)$ and $(18)$ is the direct consequence of the measurability properties of the pair $\left(\boldsymbol{U}_{t+1}, \boldsymbol{X}_{t+1}\right)$ allowing the interchange between minimization and expectation.

To recover the optimal state and control trajectories from Bellman functions, we introduce the set valued mappings:

$$
\begin{array}{ccl}
\widehat{\mathcal{S}}_{t}(R):(x, \xi) \mapsto \quad \underset{x_{t+1}}{\arg \min } & \inf _{u_{t+1}} & a_{t}^{\top} x+b_{t+1}^{\top} u_{t+1}+R\left(x_{t+1}\right), \\
& \text { s.t. } \quad & x_{t+1}=A_{t} x+B_{t+1} u_{t+1}+C_{t+1} \xi \\
& D_{t} x+E_{t+1} u_{t+1}+G_{t+1} \xi \leq 0 .
\end{array}
$$

\subsubsection{Primal SDDP}

We now apply the abstract SDDP algorithm presented in $\$ 2.3$ to the primal Bellman operator given by Equations (14). We denote, for all $t \in \llbracket 1, T \rrbracket$, and $\pi_{t}^{\xi}:=\mathbb{P}\left(\boldsymbol{\xi}_{t}=\xi\right)$ for all $\xi \in \operatorname{supp}(\boldsymbol{\xi})$. The pseudocode is given in Algorithm 2.

Remark 17. Note that, the primal Bellman operator (14) is a specialized version of the abstract Bellman operator used in Equation (10), which only involves pointwise operator in the constraints. Hence, in the forward pass we just have to compute $\widehat{\mathcal{T}}_{t}\left(\underline{V}_{t+1}^{k}\right)\left(x_{t}^{k}, \xi_{t+1}^{k}\right)$ and do not need to compute $\mathcal{T}_{t}\left(\underline{V}_{t+1}^{k}\right)\left(x_{t}^{k}\right)$ which would involve a larger linear problem. Similarly, in the backward pass at time $t$ we solve $\left|\operatorname{supp}\left(\boldsymbol{\xi}_{t+1}\right)\right|$ linear problem of the form $\widehat{\mathcal{T}}_{t}\left(\underline{V}_{t+1}^{k+1}\right)\left(x_{t}^{k}, \xi_{t+1}^{s}\right)$ (instead of the larger linear problem $\mathcal{T}_{t}\left(\underline{V}_{t+1}^{k+1}\right)\left(x_{t}^{k}\right)$ ) and then perform an expectation. We will show in the sequel that this is no more the case in the dual SDDP algorithm. 
Data: Initial point $x_{0}$, initial lower bounds $\underline{V}_{t}^{0}$ on $V_{t}$

for $k=0,1, \ldots$ do

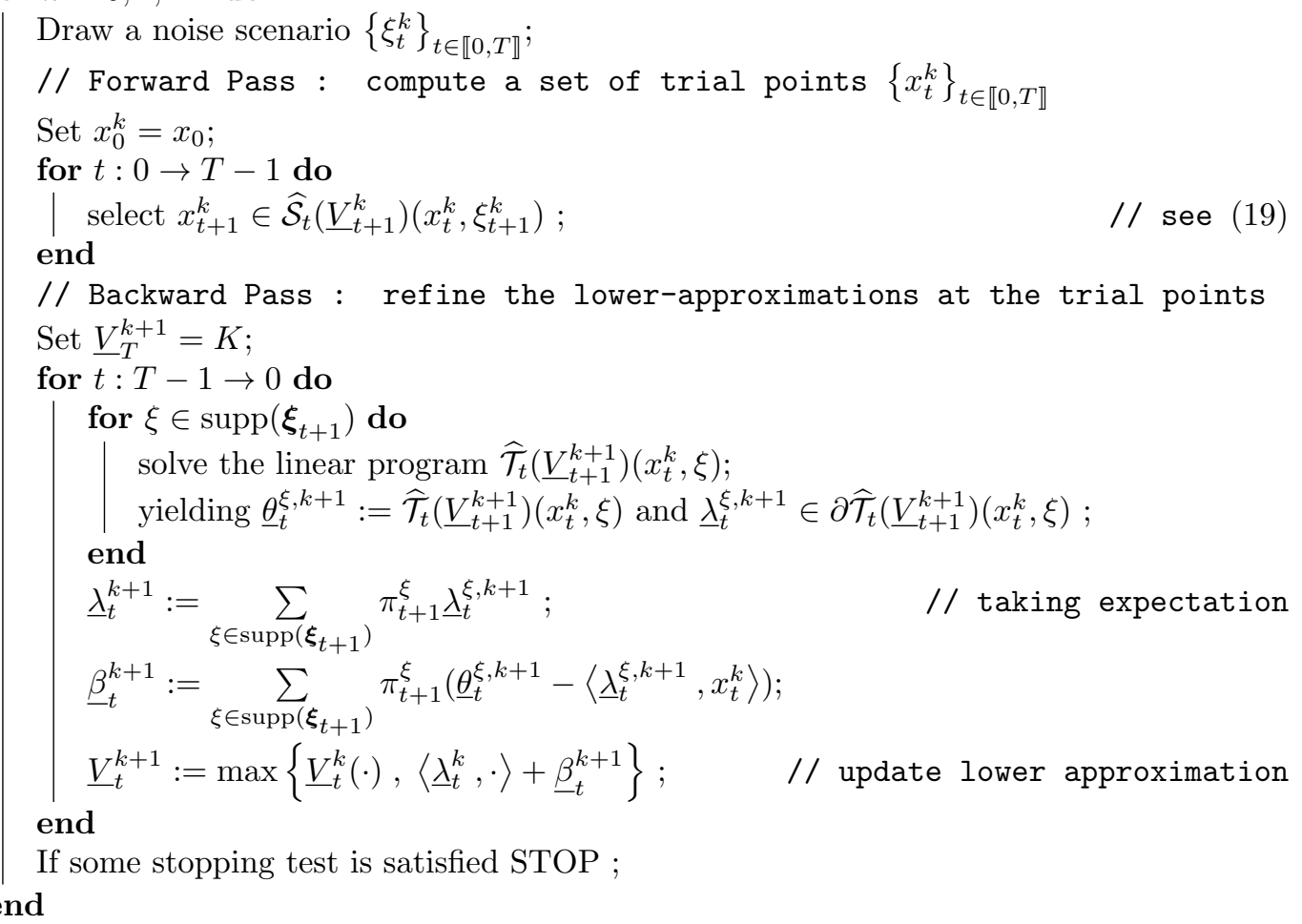

Algorithm 2: Primal SDDP algorithm

Proposition 18. Under Assumptions 1 and 2, the primal SDDP algorithm yields a converging lower bound for the value of Problem (1). Further, the strategy induced by $\underline{V}_{t}^{(k)}$ is converging toward an optimal strategy.

Proof. By Assumption 1 and Dynamic Programming, we know that the value functions $\left(V_{t}\right)_{t \in \llbracket 0, T \rrbracket}$ follow the recursion 16]. By Lemma 16, we have the compatibility of the sequence of LBOs $\left(\mathcal{T}_{t}\right)_{t \in \llbracket 0, T-1 \rrbracket}$. Further, as for any $t \in \llbracket 0, T-1 \rrbracket, \mathcal{T}_{t}$ is compact, the sequence $\left(x_{t}^{k}\right)_{k \in \mathbb{N}}$ generated by the algorithm remains in a compact. Hence, we can apply Proposition 14.

Proof of the convergence of the strategy obtained can be found in Girardeau et al. 2014.

\subsection{Dual SDDP}

We present here a dual SDDP algorithm, which leverages the conjugacy results of $\$ 2.2$. We show that the Fenchel conjugates of the primal value functions $\left(V_{t}\right)_{t \in \llbracket 0, T \rrbracket}$ follow a recursive equation on which we apply the abstract SDDP algorithm 1. 


\subsubsection{Dual Dynamic Programming equations}

By Definition 9, a straightforward computation shows that the dual LBO of $\mathcal{T}_{t}$, is given by

$$
\begin{aligned}
& \mathcal{T}_{t}^{\ddagger}(Q): \lambda_{t} \mapsto \inf _{\boldsymbol{\Lambda}_{t+1}, \boldsymbol{N}_{t+1} \geq 0} \mathbb{E}\left[-\left(C_{t+1}^{\top} \boldsymbol{\Lambda}_{t+1}+G_{t+1}^{\top} \boldsymbol{N}_{t+1}\right)^{\top} \boldsymbol{\xi}_{t+1}+Q\left(\boldsymbol{\Lambda}_{t+1}\right)\right] \\
& \text { s.t. } a_{t}+A_{t}^{\top} \mathbb{E}\left[\boldsymbol{\Lambda}_{t+1}\right]+D_{t}^{\top} \mathbb{E}\left[\boldsymbol{N}_{t+1}\right]-\lambda_{t}=0 \\
& b_{t+1}+B_{t+1}^{\top} \boldsymbol{\Lambda}_{t+1}+E_{t+1}^{\top} \boldsymbol{N}_{t+1}=0,
\end{aligned}
$$

where $\boldsymbol{\Lambda}_{t+1}: \Omega \rightarrow \mathbb{R}^{n_{x}}$ and $\boldsymbol{N}_{t+1}: \Omega \rightarrow \mathbb{R}^{n_{c}}$ are two $\boldsymbol{\xi}_{t+1}$-measurable random variables.

In Equation (20), the function $Q$ is a cost-to-go at time $t+1$ for the dual linear problem, $\lambda_{t}$ is a state variable, and $\left(\boldsymbol{\Lambda}_{t+1}, \boldsymbol{N}_{t+1}\right)$ are control variables. Equations 20b] and (20c) define the admissible control set of the problem.

Theorem 19. We assume that $K$ is a polyhedral function and that Assumption 2 holds true. For any $t \in \llbracket 0, T \rrbracket$, we denote $\mathcal{D}_{t}:=V_{t}^{\star}$, where $V_{t}$ are the Bellman value functions obtained by (16). Let, for all $t \in \llbracket 0, T \rrbracket, L_{t}>0$ be such that $V_{t}$ is $L_{t}$-Lipschitz (for the $L^{1}$-norm) on its domain. Then the sequence of dual value functions $\left\{\mathcal{D}_{t}\right\}_{t \in \llbracket 0, T \rrbracket}$ satisfies the following backward recursion:

$$
\begin{aligned}
\mathcal{D}_{T} & =K^{\star}, \\
\mathcal{D}_{t} & =\mathcal{T}_{t, L_{t+1}}^{\ddagger}\left(\mathcal{D}_{t+1}\right) \quad \forall t \in \llbracket 0, T-1 \rrbracket,
\end{aligned}
$$

where $\mathcal{T}_{t, L_{t+1}}^{\ddagger}$ is defined by Equation 20], with the additional constraint $\left\|\boldsymbol{\Lambda}_{t+1}(\omega)\right\|_{\infty} \leq L_{t+1}$.

Proof. From Lemma 16, we have that $\left\{\mathcal{T}_{t}\right\}_{t=\llbracket 0, T-1 \rrbracket}$ is a $K$-compatible sequence of compact LBOs, with the sequence $\left\{V_{t}\right\}_{t=\llbracket 0, T \rrbracket}$ of Bellman functions defined by Equation (16). Let $t \in$ $\llbracket 0, T-1 \rrbracket$. Consider the $L_{t}$-Lipschitz regularization of $V_{t}$ defined by $V_{t}^{L_{t}}:=V_{t} \square\left(L_{t}\|\cdot\|_{1}\right)$ (see A.1 for details). As $V_{t}$ is $L_{t}$-Lipschitz continuous on its domain by Proposition $8, V_{t}^{L_{t}}$ coincides with $V_{t}$ on its domain, and is $L_{t}$-Lipschitz continuous everywhere. The compatibility property implies that what only matters is the restriction of $V_{t+1}$ to $\operatorname{dom}\left(\mathcal{G}_{t+1}\right)$, and thus $V_{t}=\mathcal{T}_{t}\left(V_{t+1}^{L_{t+1}}\right)$. Theorem 10 applies, so that

$$
\left[V_{t}\right]^{\star}=\mathcal{T}_{t}^{\ddagger}\left(\left[V_{t+1}^{L_{t+1}}\right]^{\star}\right)
$$

As $V_{t+1}$ and $L_{t+1}\|\cdot\|_{1}$ takes values in $(-\infty,+\infty$ ], we have (Bauschke et al., 2017, Corollary $13.24]$ )

$$
\left[V_{t+1}^{L_{t+1}}\right]^{\star}=\left[V_{t+1}\right]^{\star}+\mathbb{I}_{B_{\infty}\left(0, L_{t+1}\right)}
$$

where $B_{\infty}\left(0, L_{t+1}\right)$ is the $L_{\infty}$-ball of radius $L_{t+1}$ centered in 0 . Thus we have

$$
\mathcal{D}_{t}=\mathcal{T}_{t}^{\ddagger}\left(\mathcal{D}_{t+1}+\mathbb{I}_{B_{\infty}\left(0, L_{t+1}\right)}\right),
$$

which precisely matches the backward recursion 21.

Remark 20. Lemma 13 shows how to find such a sequence of Lipschitz constants $\left\{L_{t}\right\}_{t \in \llbracket 0, T \rrbracket}$. But in some cases we can directly derive Lipschitz constant on the value functions, and plug it into Equation (21). 


\subsubsection{Dual SDDP}

From now on we assume that

Assumption 3. $\left\{\mathcal{T}_{t, L_{t+1}}^{\ddagger}\right\}_{t \in \llbracket 0, T-1 \rrbracket}$ is $K^{\star}$-compatible.

This is ensured for example if all $A_{t}$ in Problem (1) are square invertible matrices (see Appendix A.4.

The dual value functions $\left\{\mathcal{D}_{t}\right\}_{t \in \llbracket 0, T \rrbracket}$ are solutions of a Bellman recursion (Theorem 19 involving linear Bellman operators $\left\{\mathcal{T}_{t, L_{t+1}}^{\ddagger}\right\}_{t \in \llbracket 0, T-1 \rrbracket}$, thus opening the door to the computation of outer approximations $\left\{\underline{\mathcal{D}}_{t}^{k}\right\}_{t \in \llbracket 0, T \rrbracket}$ by SDDP, as shown in Algorithm 3

Data: Initial primal point $x_{0}$, Lipschitz bounds $\left\{L_{t}\right\}_{t \in \llbracket 0, T \rrbracket}$

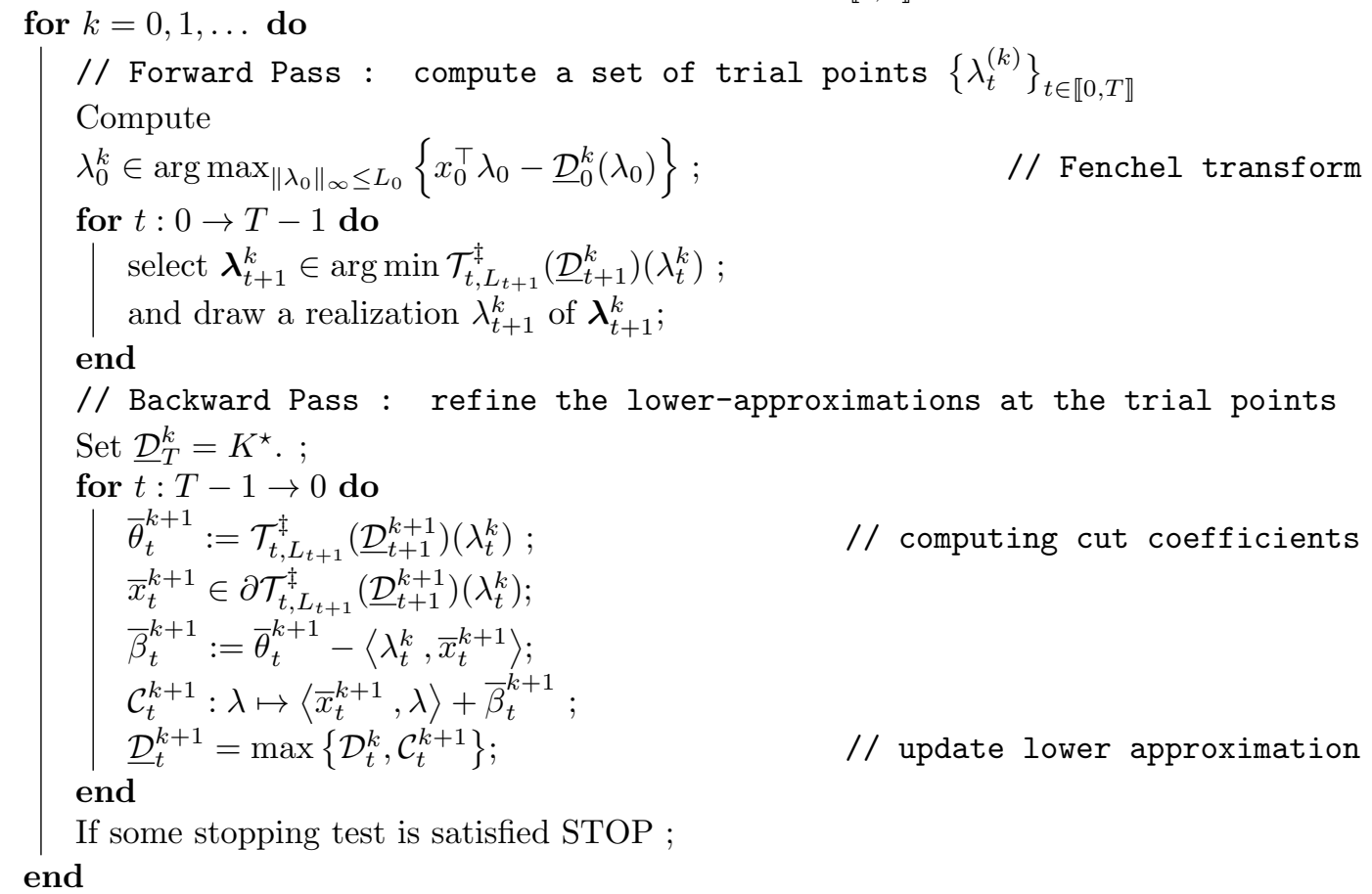

\section{Algorithm 3: Dual SDDP algorithm}

Lemma 21. For all $t \in \llbracket 0, T-1 \rrbracket,\left(\underline{D}_{t}^{k}\right)^{\star}$ is a decreasing sequence of upper bounds of the primal value function $V_{t}:\left(\underline{\mathcal{D}}_{t}^{k}\right)^{\star} \geq V_{t}$.

Proof. The sequence of functions $\underline{\mathcal{D}}_{t}^{k}$ is obtained by applying SDDP to the dual recursion (21), which is an increasing sequence of lower-bounds of the function $\mathcal{D}_{t}$ by Proposition 14 . By conjugacy property, we obtain a decreasing sequence of functions $\left(\underline{\mathcal{D}}_{t}^{k}\right)^{*}$ that are upper bounds of the function $\mathcal{D}_{t}^{*}=V_{t}$.

We have the following convergence theorem.

Theorem 22. Under assumptions 1, 2 and 3. $\left(\underline{\mathcal{D}}_{0}^{k}\right)^{\star}\left(x_{0}\right)$ is a converging upper bound to the value $V\left(x_{0}\right)$ of Problem (1), that is $\lim _{k}\left(\underline{\mathcal{D}}_{0}^{k}\right)^{\star}\left(x_{0}\right)=V_{0}\left(x_{0}\right)$. 
Proof. We add a fictive time step $t=-1$, in order to approximate the Fenchel transform of $\underline{\mathcal{D}}_{0}^{k}$ at $x_{0}$. More precisely, we define $\mathcal{T}_{-1, L_{0}}^{\ddagger}$ as follows

$$
\mathcal{T}_{-1, L_{0}}^{\ddagger}(R):=\min _{\lambda_{0}:\left\|\lambda_{0}\right\|_{\infty} \leq L_{0}}-x_{0}^{\top} \lambda_{0}+R\left(\lambda_{0}\right) .
$$

then Algorithm 3 is the abstract SDDP Algorithm 1 applied to the Bellman recursion $\mathcal{D}_{t}=$ $\mathcal{T}_{t, L_{t+1}}^{\ddagger}\left(\mathcal{D}_{t+1}\right)$ for $t \in \llbracket-1, T-1 \rrbracket$ and $\mathcal{D}_{T}=K^{\star}$. The initial point is arbitrarily set to a fixed value 0 as $\mathcal{D}_{-1}$ is constant.

We check that, by definition of $\mathcal{T}_{t, L_{t}}^{\ddagger},\left\|\lambda_{t}^{k}\right\|_{\infty} \leq L_{t}$. Further, as $V_{0}$ is $L_{0}$ Lipschitz for the $L_{1}$-norm, $\max _{\lambda} x_{0}^{T} \lambda-V_{0}^{\star}(\lambda)$ is attained for $\lambda_{0}$ such that $\left\|\lambda_{0}\right\|_{\infty} \leq L_{0}$, thus we have $\mathcal{D}_{-1}(0)=$ $-[V]^{\star \star}\left(x_{0}\right)=-V\left(x_{0}\right) \in \mathbb{R}$. Finally, note that $\left\{\mathcal{T}_{t, L_{t+1}}^{\ddagger}\right\}_{t \in \mathbb{I}-1, T \rrbracket}$ is a $K^{\star}$-compatible sequence of LBO.

Lemma 21 shows that, for any $k \in \mathbb{N},-\underline{\mathcal{D}}_{-1}^{k}(0)=\left[\underline{\mathcal{D}}_{0}\left(x_{0}\right)\right]^{\star}$ is an upper bound of $V_{0}\left(x_{0}\right)$. Finally, the convergence of the abstract SDDP algorithm and the lower semi-continuity of $V_{0}$ at $x_{0}$ yields the convergence of the upper bound.

Remark 23. Recall that, in order to obtain an upper bound of the optimal value of Problem (1), the seminal method consists in computing the expected cost of SDDP's strategy with a MonteCarlo approach (see discussion in $\$ 1.2$. This approach has two weaknesses: it requires a large number $M$ of forward pass (simulation), and the bound obtained is only an upper bound with (asymptotic) probability $\alpha$, where increasing $\alpha$ increases the bound as well. Furthermore, the statistical upper-bound is not converging toward the actual problem value, unless we also increase the number of Monte-Carlo simulations along the iterations.

In contrast to the Monte Carlo method, Theorem 22 show that we obtain a converging sequence of exact upper bounds for Problem (1).

Remark 24. In the forward pass of Algorithm 3 , we need to solve $\mathcal{T}_{t, L_{t+1}}^{\ddagger}\left(\underline{\mathcal{D}}_{t+1}^{k}\right)\left(\lambda_{t}^{k}\right)$, which in extended form reads

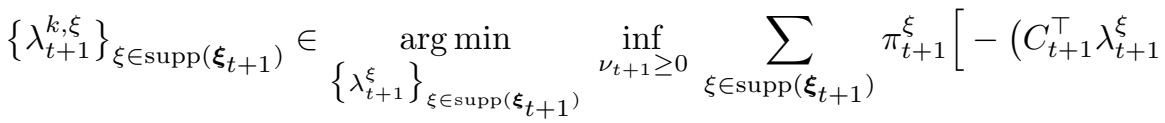

$$
\begin{aligned}
& \left.\left.+G_{t+1}^{\top} \nu_{t+1}^{\xi}\right)^{\top} \xi_{t+1}^{\xi}+\underline{\mathcal{D}}_{t+1}^{k}\left(\lambda_{t+1}^{\xi}\right)\right] \\
& \text { s.t. } \quad \sum_{\xi \in \operatorname{supp}\left(\xi_{t+1}\right)} \pi_{t+1}^{\xi}\left(A_{t}^{\top} \lambda_{t+1}^{\xi}+D_{t+1}^{\top} \nu_{t+1}^{\xi}\right)=\lambda_{t}^{k} \\
& c_{t+1}+B_{t+1}^{\top} \lambda_{t+1}^{\xi}+E_{t+1}^{\top} \nu_{t+1}^{\xi}=0 \quad \forall \xi, \\
& \left\|\lambda_{t+1}^{\xi}\right\|_{\infty} \leq L_{t+1} \text {. }
\end{aligned}
$$

Then drawing a random realization of $\boldsymbol{\lambda}_{t+1}^{k}$ consists in drawing $\xi$ with respect to the law of $\boldsymbol{\xi}_{t+1}$ and selecting $\lambda_{t+1}^{k, \xi}$.

In contrast with primal SDDP algorithm (see Remark 17), here we need to solve a linear problem coupling all possible outcomes of the random variable $\boldsymbol{\xi}_{t+1}$, both in the forward and in the backward pass. In particular it means that we can also compute cuts during the forward pass, thus rendering the backward pass optional. 


\section{Inner-approximation strategy}

In Sect. 3 we detailed how to use the SDDP algorithm to get an outer approximation $\left\{\underline{\mathcal{D}}_{t}\right\}_{t \in \llbracket 0, T \rrbracket}$ of the dual value functions $\left\{\mathcal{D}_{t}\right\}_{t \in \llbracket 0, T \rrbracket}$. We now explain how to build an inner approximation of the primal value functions $\left\{V_{t}\right\}_{t \in \llbracket 0, T \rrbracket}$ using this dual outer approximation. Assume that, $\left\{L_{t}\right\}_{t \in \llbracket 0, T-1 \rrbracket}$ is given by Lemma 13

\subsection{Inner approximation of value functions}

Let $\left\{\underline{\mathcal{D}}_{t}^{k}\right\}_{t \in \llbracket 0, T \rrbracket}$ be the outer approximation of the dual value functions $\left\{\mathcal{D}_{t}\right\}_{t \in \llbracket 0, T \rrbracket}$ obtained at iteration $k$ of the dual SDDP algorithm. We denote by $\left\{\left(\bar{x}_{t}^{\kappa}, \bar{\beta}_{t}^{\kappa}\right)\right\}_{\kappa \in \llbracket 1, k \rrbracket}$ the cuts coefficients computed by the dual SDDP algorithm:

$$
\begin{aligned}
\underline{\mathcal{D}}_{t}^{k}(\lambda)=\min _{\theta} & \theta, \\
\text { s.t. } & \theta \geq\left\langle\bar{x}_{t}^{\kappa}, \lambda\right\rangle+\bar{\beta}_{t}^{\kappa} \quad \forall \kappa \in \llbracket 1, k \rrbracket .
\end{aligned}
$$

We define the linear inner approximation $\bar{V}_{t}^{k}$ of the primal value functions $\left\{V_{t}\right\}_{t \in \llbracket 0, T \rrbracket}$ as the Lipschitz regularization of the Fenchel conjugate of the dual outer approximation.

Definition 25. We define $\bar{V}_{t}^{k}$ by

$$
\bar{V}_{t}^{k}=\left[\underline{\mathcal{D}}_{t}^{k}\right]^{\star} \square\left(L_{t}\|\cdot\|_{1}\right) \quad \forall t \in \llbracket 0, T \rrbracket .
$$

From proposition 33 in appendix we have the following properties of $\bar{V}_{t}^{k}$.

Proposition 26. For all $t \in \llbracket 0, T-1 \rrbracket$ we have

i) $\bar{V}_{t}^{k} \geq V_{t}$ on $X_{t}$.

ii) We have

$$
\begin{gathered}
\bar{V}_{t}^{k}(x)=\min _{y \in \mathbb{R}^{n_{x}}, \sigma \in \Delta} L_{t}\|x-y\|_{1}-\sum_{\kappa=1}^{k} \sigma_{\kappa} \bar{\beta}_{t}^{\kappa} \\
\text { s.t. } \sum_{\kappa=1}^{k} \sigma_{\kappa} \bar{x}_{t}^{\kappa}=y,
\end{gathered}
$$

where $\Delta=\left\{\sigma \in \mathbb{R}^{k} \mid \sigma \geq 0, \sum_{\kappa=1}^{k} \sigma_{\kappa}=1\right\}$ is the simplex of $\mathbb{R}^{k}$.

iii) The inner approximation can be computed by solving

$$
\begin{array}{rlr}
\bar{V}_{t}^{k}(x)=\sup _{\lambda, \theta} & x^{\top} \lambda-\theta & \\
\text { s.t. } & \theta \geq\left\langle\bar{x}_{t}^{i}, \lambda\right\rangle+\bar{\beta}_{t}^{\kappa} & \\
& \|\lambda\|_{\infty} \leq L_{t} . &
\end{array}
$$

iv) The Fenchel transform of the inner approximation is given by $\left[\bar{V}_{t}^{k}\right]^{\star}=\underline{\mathcal{D}}_{t}^{k}+\mathbb{I}_{B_{\infty}\left(0, L_{t}\right)}$. 
Proof. i) Lemma 21 proves that $\left[\underline{\mathcal{D}}_{t}^{k}\right]^{\star} \geq V_{t}$ for all $t \in \llbracket 0, T \rrbracket$. Thus $\bar{V}_{t}^{k} \geq V_{t} \square\left(L_{t}\|\cdot\|_{1}\right)$, which is equal to $V_{t}$ on $X_{t}$ as $V_{t}$ is $L_{t}$-Lipschitz on its domain.

ii) Further, the Fenchel conjugate $\left[\underline{\mathcal{D}}_{t}^{k}\right]^{\star}$ reads

$$
\begin{aligned}
{\left[\underline{\mathcal{D}}_{t}^{k}\right]^{\star}(x)=\sup _{\lambda, \theta} x^{\top} \lambda-\theta } & \\
& \text { s.t. } \theta \geq\left\langle\bar{x}_{t}^{i}, \lambda\right\rangle+\bar{\beta}_{t}^{\kappa} \quad \forall \kappa \in \llbracket 1, k \rrbracket,
\end{aligned}
$$

which is a linear program admitting an admissible solution, hence by strong duality

$$
\begin{aligned}
{\left[\underline{\mathcal{D}}_{t}^{k}\right]^{\star}(x)=\min _{\sigma \in \Delta}-\sum_{\kappa=1}^{k} \sigma_{\kappa} \bar{\beta}_{t}^{\kappa} } \\
\text { s.t. } \sum_{\kappa=1}^{k} \sigma_{\kappa} \bar{x}_{t}^{\kappa}=x .
\end{aligned}
$$

Taking the inf-convolution with $L_{t}\|\cdot\|$ yields Problem 25

iii) The right hand side of Equation $(26)$ is simply $\left[\underline{\mathcal{D}}_{t}^{k}+\mathbb{I}_{B_{\infty}\left(0, L_{t}\right)}\right]^{\star}(x)$, which is equal to $\left[\underline{\mathcal{D}}_{t}^{k}\right]^{\star} \square\left[\mathbb{I}_{B_{\infty}\left(0, L_{t}\right)}\right]^{\star}(x)$ by finite polyhedrality, hence the results.

iv) Finally,

$$
\left[\bar{V}_{t}^{k}\right]^{\star}=\left[\underline{\mathcal{D}}_{t}^{k}\right]^{\star \star}+\mathbb{I}_{B_{\infty}\left(0, L_{t}\right)}
$$

Figure 4.1 illustrates how to interpret the dual outer approximation as a primal inner approximation of the original value function (in black). The slopes $x_{1}, x_{2}, x_{3}$ computed for the dual outer approximation (blue curve, right) are breakpoints for the primal problem and we can consider the value of the dual value function at these points to build a primal inner approximation (blue curve, left).

\subsection{A bound on the inner approximation strategy value}

Hence, we have obtained inner approximations of the primal value functions. Such approximations can be used to define an admissible strategy for the initial problem. We now study the properties of such a strategy.

Lemma 27. We have,

$$
\mathcal{T}_{t, L}^{\ddagger}\left(\underline{\mathcal{D}}_{t+1}^{k}\right) \geq \underline{\mathcal{D}}_{t}^{k} \quad \forall t \in \llbracket 0, T \rrbracket .
$$

Proof. Equation (27) is satisfied for $k=0$. Assume that Equation (27) holds at iteration $k$. By definition of $\mathcal{C}^{k+1}$ in Algorithm 3 , we have $\mathcal{C}^{k+1} \leq \mathcal{T}_{t, L_{t+1}}^{\ddagger}\left(\underline{\mathcal{D}}_{t+1}^{k+1}\right)$. On the other hand, by monotonicity of $\mathcal{T}_{t, L_{t+1}}^{\ddagger}$, since $\underline{\mathcal{D}}_{t+1}^{k+1} \geq \underline{\mathcal{D}}_{t+1}^{k}$, we have $\mathcal{T}_{t, L_{t+1}}^{\ddagger}\left(\underline{\mathcal{D}}_{t+1}^{k+1}\right) \geq \mathcal{T}_{t, L_{t+1}}^{\ddagger}\left(\underline{\mathcal{D}}_{t+1}^{k}\right)$ which is greater than $\underline{\mathcal{D}}_{t}^{k}$ by induction hypothesis. Thus, $\mathcal{T}_{t, L_{t+1}}^{\ddagger}\left(\underline{\mathcal{D}}_{t+1}^{k+1}\right) \geq \max \left\{\underline{\mathcal{D}}_{t}^{k}, \mathcal{C}^{k+1}\right\}=\underline{\mathcal{D}}_{t}^{k+1}$.

Lemma 28. Let $\bar{V}_{t}^{k}$ be the inner approximation of the value function $V_{t}$ generated at iteration $k$ of the dual SDDP algorithm. Then,

$$
\mathcal{T}_{t}\left(\bar{V}_{t+1}^{k}\right)(x) \leq \bar{V}_{t}^{k}(x) \quad \forall t \in \llbracket 0, T \rrbracket .
$$


Primal

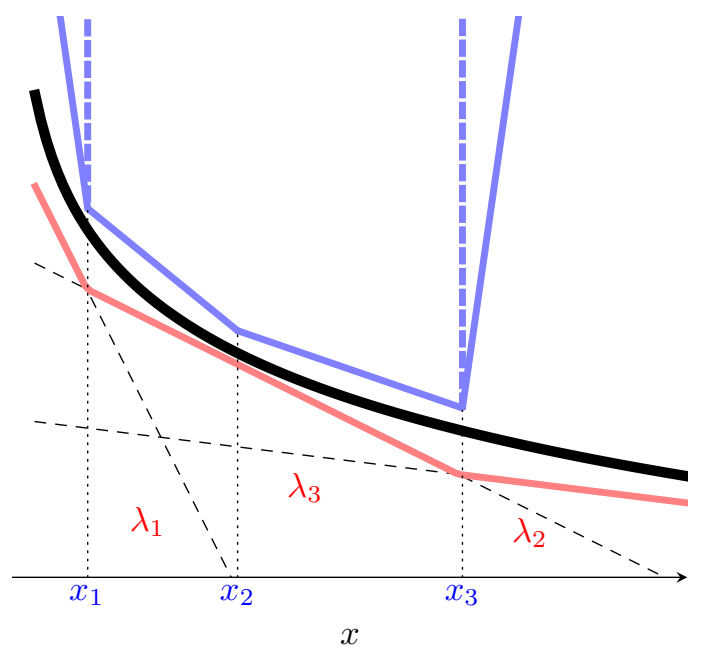

Dual

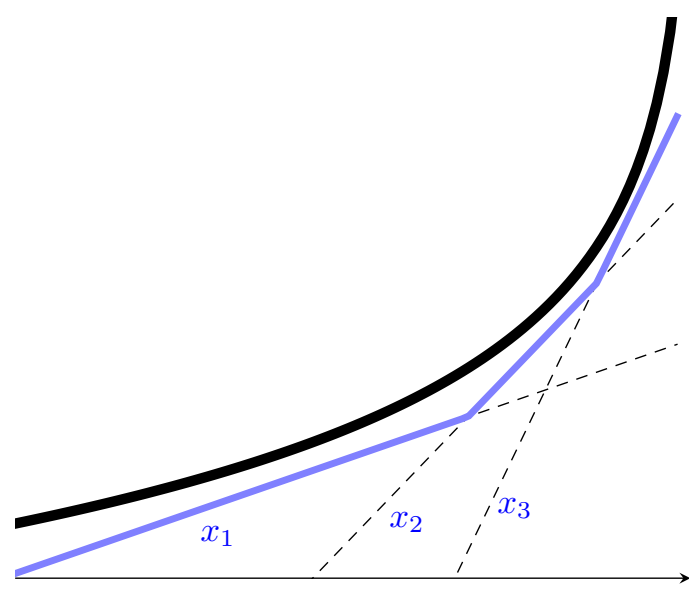

$\lambda$

Figure 1: Primal SDDP computes an outer approximation (in red) of the original value function (in black). Dual SDDP computes an outer approximation in the dual, whose (regularized) Fenchel-transform (in blue) yields an inner approximation of the primal problem.

Proof. We have

$$
\begin{aligned}
{\left[\mathcal{T}_{t}\left(\bar{V}_{t+1}^{k}\right)\right]^{\star} } & =\mathcal{T}_{t}^{\ddagger}\left(\left[\bar{V}_{t+1}^{k}\right]^{\star}\right) \\
& =\mathcal{T}_{t}^{\ddagger}\left(\underline{\mathcal{D}}_{t+1}^{k}+\mathbb{I}_{B \infty}\left(0, L_{t+1}\right)\right) \\
& =\mathcal{T}_{t, L}^{\ddagger}\left(\underline{\mathcal{D}}_{t+1}^{k}\right) \\
& \geq \underline{\mathcal{D}}_{t}^{k}
\end{aligned}
$$

by Proposition 26

by Lemma 27

Furthermore, as $\mathcal{T}_{t}\left(\bar{V}_{t+1}^{k}\right)$ is polyhedral, we have

$$
\mathcal{T}_{t}\left(\bar{V}_{t+1}^{k}\right)=\left[\mathcal{T}_{t}\left(\bar{V}_{t+1}^{k}\right)\right]^{\star \star} \leq\left[\underline{\mathcal{D}}_{t}^{k}\right]^{\star}
$$

and as $\bar{V}_{t+1}^{k}$ is $L_{t+1}$-Lipschtiz, then $\mathcal{T}_{t}\left(\bar{V}_{t+1}^{k}\right)$ is $L_{t}$-Lipschtiz, thus $\mathcal{T}_{t}\left(\bar{V}_{t+1}^{k}\right) \leq\left[\underline{\mathcal{D}}_{t}^{k}\right]^{\star} \square\left(L_{t}\|\cdot\|\right)$ which ends the proof.

We are now able to state the main result of this section.

Theorem 29. Let $\left\{\boldsymbol{X}_{t}^{I A}, \boldsymbol{U}_{t}^{I A}\right\}_{t \in \llbracket 0, T-1 \rrbracket}$ be the state and control processes obtained by applying the strategy induced by the inner approximation $\left\{\bar{V}_{t}^{k}\right\}_{t \in \llbracket 0, T \rrbracket}$, that is, $\left(\boldsymbol{X}_{t+1}^{I A}, \boldsymbol{U}_{t+1}^{I A}\right) \in$ $\mathcal{S}_{t}\left(\bar{V}_{t+1}^{k}\right)\left(\boldsymbol{X}_{t}^{I A}\right)$. Consider the expected cost of this strategy when starting from state $x$ at time $t$ :

$$
C_{t}^{I A}(x)=\mathbb{E}\left(\sum_{\tau=t}^{T-1} a_{\tau}^{\top} \boldsymbol{X}_{\tau}^{I A}+b_{\tau+1}^{\top} \boldsymbol{U}_{\tau+1}^{I A}+K\left(\boldsymbol{X}_{T}^{I A}\right) \mid \boldsymbol{X}_{t}^{I A}=x\right) .
$$

Then,

$$
C_{t}^{I A}(x) \leq \bar{V}_{t}^{k}(x)
$$


Further, the inner approximation strategy is converging in the sense that $\lim _{k \rightarrow+\infty} C_{0}^{I A, k}\left(x_{0}\right)=$ $V_{0}\left(x_{0}\right)$.

Proof. We proceed by backward induction on time $t$. The property holds for $t=T$.

Assume that $C_{t+1}^{I A} \leq \bar{V}_{t+1}^{k}$. We have

$$
\begin{aligned}
C_{t}^{I A}(x) & =\mathbb{E}\left[a_{t}^{\top} x+b_{t+1}^{\top} \boldsymbol{U}_{t+1}^{I A}+C_{t+1}^{I A}\left(\boldsymbol{X}_{t+1}^{I A}\right)\right] \\
& \leq \mathbb{E}\left[a_{t}^{\top} x+b_{t+1}^{\top} \boldsymbol{U}_{t+1}^{I A}+\bar{V}_{t+1}^{k}\left(\boldsymbol{X}_{t+1}^{I A}\right)\right] \\
& =\mathcal{T}_{t}\left(\bar{V}_{t+1}^{k}\right)(x) \\
& \leq \bar{V}_{t}^{k}(x)
\end{aligned}
$$

by induction

by definition of $\boldsymbol{U}_{t+1}^{I A}$

by Lemma 28

hence the result.

Finally, the convergence of the strategy is easily obtained. By definition of $V_{0}\left(x_{0}\right)$, we have $C_{0}^{I A, k}\left(x_{0}\right) \geq V_{0}\left(x_{0}\right)$. Furthermore, $V_{0}\left(x_{0}\right) \leq C_{0}^{I A, k}\left(x_{0}\right) \leq \bar{V}_{0}^{k}\left(x_{0}\right)$. By Theorem 22 , we know that $\lim _{k}\left(\bar{V}_{0}^{k}\right)\left(x_{0}\right)=V_{0}\left(x_{0}\right)$. Hence the result.

Remark 30. A similar result on the performance of an inner approximation is given in Philpott et al. 2013. As already explained in $\$ 1.2$, the authors construct polyhedral inner approximations $V_{t}$ of the Bellman functions $V_{t}$. They then prove that the expected cost of the policy based on the functions $\bar{V}_{t}$ is always less than or equal to the deterministic upper bound given by the inner approximation algorithm.

We sum up the available inequalities for the values obtained when implementing the primal and dual SDDP algorithms.

$$
\begin{aligned}
& \underline{V}_{0}\left(x_{0}\right) \leq V_{0}\left(x_{0}\right) \leq \bar{V}_{0}\left(x_{0}\right) \\
& \underline{V}_{0}\left(x_{0}\right) \leq C_{0}^{I A}\left(x_{0}\right) \leq \bar{V}_{0}\left(x_{0}\right) \\
& \underline{V}_{0}\left(x_{0}\right) \leq C_{0}^{O A}\left(x_{0}\right)
\end{aligned}
$$

Equation (30a) corresponds to the deterministic bounds of the optimal value of Problem (1), whereas Equations (30b) and (30c) are of statistical nature.

\section{$5 \quad$ Numerical results}

In this section, we present some numerical results applying dual SDDP and inner strategy evaluation to a stochastic operation planning problem inspired by Électricité de France (EDF, main European electricity producer). The problem is about the energy production planning on a multiperiod horizon including a network of production zones, like in the European Market for electricity. It results in a large-scale stochastic multi-stage optimization problem, for which we need to determine strategies for the management of the European water dams. Such strategies cannot be computed via Dynamic Programming because of the state variable size, so that SDDP is the reference method to compute the optimal Bellman functions.

\subsection{Description of the problem}

We consider an operation planning problem at the European scale. Different countries are connected together via a network, and exchange energy with their neighbors. We formulate the 


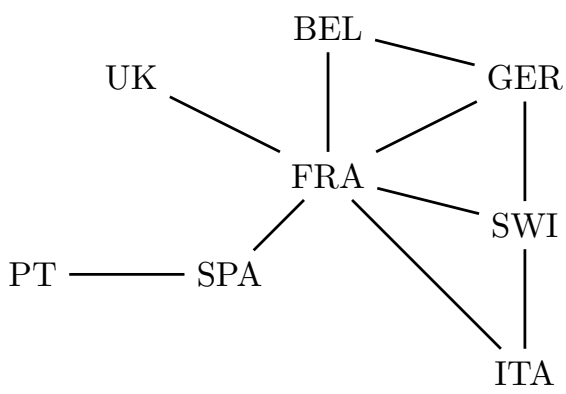

Figure 2: Schematic description of the European network

problem on a graph, where each country is modeled as a node and each interconnection line between two countries as an edge (see Figure 2). Every country uses a reservoir to store energy, and must fulfill its own energy demand. To do so, it can produce energy from its reservoir, with its local thermal power plant, or it can import energy from the other countries. A very similar problem has already been studied by Mahey et al. [2017]. Its formulation is close to the one given in Shapiro et al. 2012 concerning the Brazilian interconnected power system.

Let $\mathcal{G}=(\mathcal{N}, \mathcal{E})$ the graph modeling the European network. The number of nodes in $\mathcal{N}$ is denoted by $n$ and the number of edges in $\mathcal{E}$ by $\ell$. For each node $i \in \llbracket 1, n \rrbracket$, we denote by $v_{t}^{i}$ the energy stored in the reservoir at time $t$. The reservoir's dynamics is given by

$$
v_{t+1}^{i}=v_{t}^{i}+a_{t+1}^{i}-q_{t+1}^{i}-s_{t+1}^{i}
$$

where $a_{t+1}^{i}$ is the (random) water inflow in the reservoir and $q_{t+1}^{i}$ is the water turbinated between time $t$ and $t+1$ in order to produce electricity. We add a spillage $s_{t+1}^{i}$ as recourse variable to avoid to overthrow the reservoir. Still at node $i$, the load balance equation at stage $t$ writes

$$
q_{t}^{i}+g_{t}^{i}+\sum_{j \in N_{i}} f_{t}^{j i}+r_{t}^{i}=d_{t}^{i}
$$

where $g_{t}^{i}$ is the thermal production, $N_{i} \subset \mathcal{N}$ is the set of nodes connected to node $i$ and $f_{t}^{j i}$ is the energy exchanged between nodes $j \in N_{i}$ and node $i, d_{t}^{i}$ is the (random) demand of the node and $r_{t}^{i} \geq 0$ is a recourse variable added to ensure that the load balance is always satisfied. The thermal production $g_{t}^{i}$ and the exchanges $f_{t}^{j i}$ between node $i$ and nodes $j \in N_{i}$ induce linear costs, and the cost of the recourse variable $r_{t}^{i}$ is taken into account through a linear penalization. Hence the total cost attached to node $i$ at time $t$ writes

$$
c_{t}^{i} g_{t}^{i}+\delta_{t}^{i} r_{t}^{i}+\sum_{j \in N_{i}} p_{t}^{j i} f_{t}^{j i}
$$

where $c_{t}^{i}$ is the thermal price, $\delta_{t}^{i}$ is the recourse price and $p_{t}^{j i}$ is the transportation price between nodes $j$ and $i$. To avoid empty stocks at the end of the time horizon, we penalize the final stock at each node $i$ if it is beyond a threshold $v_{0}^{i}$ using a piecewise linear function:

$$
K^{i}\left(v_{T}^{i}\right)=\kappa_{T}^{i} \max \left(0, v_{0}^{i}-v_{T}^{i}\right) .
$$

Stocks and controls are bounded:

- $0 \leq v_{t}^{i} \leq \bar{v}^{i}$, reservoir volume upper bound, 
- $0 \leq q_{t}^{i} \leq \bar{q}^{i}$, reservoir generation upper bound,

- $0 \leq g_{t}^{i} \leq \bar{g}^{i}$, thermal generation upper bound,

- $0 \leq r_{t}^{i}$, recourse control lower bound,

- $\underline{f}^{j i} \leq f_{t}^{j i} \leq \bar{f}^{j i}$, energy flow lower and upper bound.

This problem is formulated as a stochastic optimal control problem, where for all $t$,

- the state is $v_{t}=\left(v_{t}^{1}, \cdots, v_{t}^{n}\right)\left(\right.$ denoted $x_{t}$ in $\S 3$,

- the control is $u_{t}=\left(q_{t}, s_{t}, g_{t}, r_{t}, f_{t}\right)$, with $q_{t}=\left(q_{t}^{i}\right)_{i \in \llbracket 1, n \rrbracket}$ and likewise for $s_{t}, g_{t}, r_{t}$ and $f_{t}$,

- the uncertainty is $\xi_{t}=\left(a_{t}^{i}, d_{t}^{i}\right)_{i \in \llbracket 1, n \rrbracket}$.

The state has dimension $n$, the control $u_{t}$ dimension $4 n+\ell$ and the uncertainty $\xi_{t}$ dimension $2 n$. We assume that the random variables $\boldsymbol{\xi}_{t}$ have a discrete finite support. For a given realization $\left(a_{t+1}, d_{t+1}\right)$ of the uncertainty, the primal Bellman operator $\widehat{\mathcal{T}}_{t}$, defined by $(18)$, writes

$$
\begin{aligned}
& \widehat{\mathcal{T}}_{t}\left(V_{t+1}\right)\left(v_{t},\left(a_{t+1}, d_{t+1}\right)\right)= \min _{v_{t+1}, q_{t+1}, s_{t+1}, g_{t+1}, r_{t+1}, f_{t+1}} \sum_{i=1}^{n}\left(c_{t+1}^{i} g_{t+1}^{i}+\delta_{t+1}^{i} r_{t+1}^{i}+\right. \\
&\left.\sum_{j \in N_{i}} p_{t+1}^{j i} f_{t+1}^{j i}\right)+V_{t+1}\left(v_{t+1}\right), \\
& \text { s.t. } v_{t+1}^{i}=v_{t}^{i}-q_{t+1}^{i}-s_{t+1}^{i}+a_{t+1}^{i}, \\
& q_{t+1}^{i}+g_{t+1}^{i}+\sum_{j \in N_{i}} f_{t+1}^{j i}+r_{t+1}^{i}=d_{t+1}^{i}, \\
& 0 \leq v_{t+1}^{i} \leq \bar{v}^{i}, 0 \leq q_{t+1}^{i} \leq \bar{q}^{i}, 0 \leq r_{t+1}^{i} \\
& 0 \leq g_{t+1}^{i} \leq \bar{g}^{i}, \underline{f}^{j i} \leq f_{t+1}^{j i} \leq \bar{f}^{j i} .
\end{aligned}
$$

We rewrite Problem (35) in the standard form (1) with matrices $(A, B, C)$ for the dynamics and $(D, E, G)$ for the constraints. We note that $A$ is the identity matrix $I_{n}$. The expressions of these matrices are given in $\$$ A.2. Then the expression 20 of the dual Bellman operator $\mathcal{T}_{t}^{\ddagger}$ is obtained in a straightforward manner, namely

$$
\begin{aligned}
& \mathcal{T}_{t, L_{t+1}}^{\ddagger}\left(\mathcal{D}_{t+1}\right)\left(\lambda_{t}\right)=\inf _{\lambda_{t+1}^{\xi}, \nu_{t+1}^{\xi} \geq 0} \sum_{\xi \in \operatorname{supp}\left(\boldsymbol{\xi}_{t+1}\right)} \pi_{\xi}\left[-\left(\xi_{t+1}^{\xi}\right)^{\top} C^{\top} \lambda_{t+1}^{\xi}-g_{t+1}^{\top} \nu_{t+1}^{\xi}+\mathcal{D}_{t+1}\left(\lambda_{t+1}^{\xi}\right)\right] \\
& \text { s.t. } \sum_{\xi \in \operatorname{supp}\left(\xi_{t+1}\right)} \pi_{\xi}\left(\lambda_{t+1}^{\xi}+D^{\top} \nu_{t+1}^{\xi}\right)=\lambda_{t} \\
& \bar{c}_{t+1}+B^{\top} \lambda_{t+1}^{\xi}+E^{\top} \nu_{t+1}^{\xi}=0 \quad \forall \xi \\
&\left\|\lambda_{t+1}^{\xi}\right\|_{\infty} \leq L_{t+1}
\end{aligned}
$$

where $L_{t}$ a Lipschitz constant of $V_{t+1}$. 


\subsection{Numerical implementation}

The forward and backward passes of dual SDDP are independent from the forward and backward passes of primal SDDP. Accordingly, a first "natural" implementation of the whole algorithm runs primal and dual SDDP in two independent processes, and thus enables to compute primal and dual value functions in parallel.

However, each backward pass of the primal SDDP algorithm computes a set of cuts whose slopes are $\left\{\lambda_{t}\right\}_{t \in \llbracket 0, T \rrbracket}$. As explained in Figure 4.1, these slopes can be considered as trajectories for the dual problem. If primal SDDP has converged, they are even the optimal co-state of the problem, because of the Fenchel-Young equality. Thereby, it may prove useful to view these sequences of slopes as trajectories for the dual problem, along which we run afterward a backward pass producing cuts for the dual problem. In this implementation, each iteration of the algorithm consists of four steps (the complete algorithm is given in A.3):

1. Run a forward pass of primal SDDP Algorithm 2 and get trajectories $\left\{x_{t}\right\}_{t \in \llbracket 0, T \rrbracket}$.

2. Run a backward pass of primal SDDP Algorithm 2 along $\left\{x_{t}\right\}_{t \in \llbracket 0, T \rrbracket}$ and obtain new slopes $\left\{\lambda_{t}\right\}_{t \in \llbracket 0, T \rrbracket}$.

3. Run a backward pass of dual SDDP Algorithm 3 along $\left\{\lambda_{t}\right\}_{t \in \llbracket 0, T \rrbracket}$, thus updating the sets of cuts for the dual problem.

4. Run a forward pass of dual SDDP Algorithm 3 and update the cuts along the obtained trajectories.

The last step of this iteration ensures that we recover the convergence hypotheses of SDDP, as given in Girardeau et al. [2014], by having one set of cuts computed at point sampled along uncertainty drawn independently from the past. This algorithm has the same number of forward and backward passes as the original one (one forward pass and one backward pass in both the primal and the dual space). However, this scheme proves to be numerically more efficient, both in term of convergence and computation time. That is why we use this implementation in all the numerical experiments.

From the computational point of view, we implement primal and dual SDDP in Julia 0.6, with the StochDynamicProgramming. $j 1$ package built on top of the JuMP modeler of Dunning et al. 2017]. We use Gurobi 7.02 to solve the LP subproblems. All experiments are run on a Intel Core i7-5500 CPU @2.4GHz, 64bit computer.

\section{$5.3 \quad$ Results}

We consider the problem described at $\$ 5.1$, which exhibits a 8-dimensional state. We aim to compute the value functions $\left\{V_{t}\right\}_{t \in \llbracket 0, T \rrbracket}$ with monthly time steps, and we consider different time horizons $T$, depending on the desired goal (illustration of convergence, comparison of bounds... ). The uncertainties in the model are the inflows $a_{t}$ in the reservoir and the demands $d_{t}$ in every considered countries. Inflows and demands trajectories are simulated using a software provided by EDF, so that these data are realistic enough. From these simulated samples, we use quantization methods to obtain the marginal laws of the uncertainty $\boldsymbol{\xi}_{t}$ at each $t \in \llbracket 0, T \rrbracket$. The support of the quantized probability laws is limited to 10 possible values for $\boldsymbol{\xi}_{t}$ at each timestep $t$.

To solve the problem, we run primal and dual SDDP on 1,000 iterations, with a single forward pass in the primal and in the dual. 


\subsubsection{Assessing convergence}

To ease the description of the results, we denote by Primal LB the primal lower bound $\underline{V}_{0}\left(x_{0}\right)$ obtained by primal SDDP, and by Dual UB the upper bound $\left[\underline{\mathcal{D}}_{0}\right]^{\star}\left(x_{0}\right)$ given by dual SDDP. The Monte Carlo cost evaluation obtained by simulating the outer (resp. inner) strategy uses the procedure described in 33.1 .2 and is denoted by MC OA (resp. MC IA). Confidence intervals (with a confidence level $\alpha=97.5 \%$ ) are associated to these Monte Carlo approximations, and we denote by MC OA UB and MC IA UB the associated upper bounds of these intervals. Whereas Primal LB and Dual UB are deterministic bounds, MC OA, MC IA MC OA UB and MC IA UB are statistical quantities.

Solving the problem over a one-year time horizon. First, we run dual and primal SDDP on a twelve months problem, that is, with $T=12$. Convergence of the optimal costs given by dual and primal SDDP is detailed in Figure 3. The two last columns in the table give the cumulative

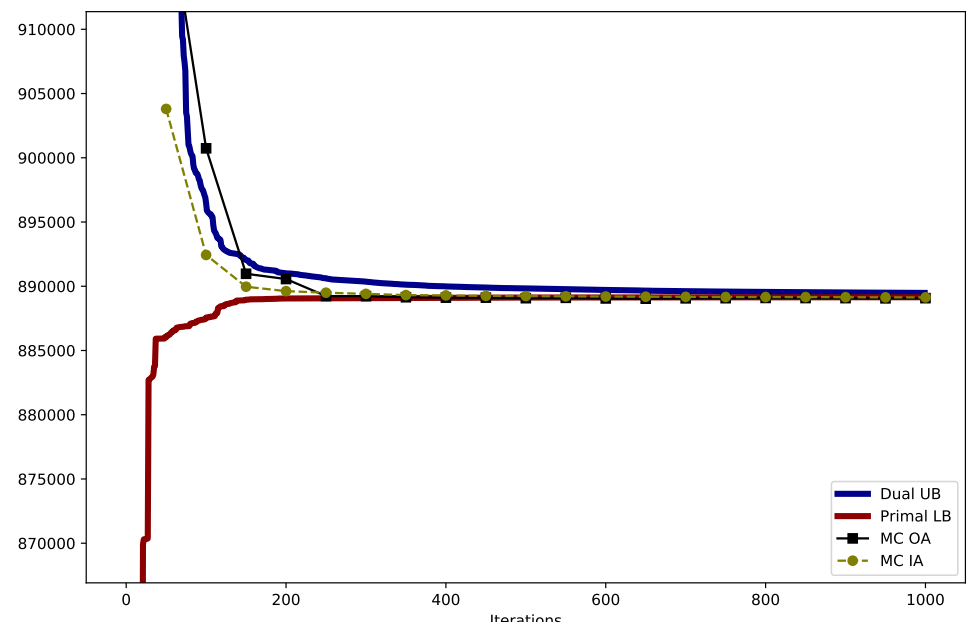

\begin{tabular}{cccccc}
\hline Iter. & Primal LB $\left(\times 10^{5}\right)$ & Dual UB $\left(\times 10^{5}\right)$ & Gap $(\%)$ & Time LB $(\mathrm{s})$ & Time UB (s) \\
\hline 50 & 8.861 & 9.577 & 8.08 & 2. & 8. \\
100 & 8.874 & 8.969 & 1.06 & 3. & 22. \\
200 & 8.890 & 8.910 & 0.21 & 8. & 72. \\
300 & 8.891 & 8.904 & 0.14 & 13. & 153. \\
400 & 8.891 & 8.900 & 0.09 & 20. & 275. \\
500 & 8.891 & 8.898 & 0.08 & 29. & 443. \\
600 & 8.891 & 8.897 & 0.06 & 38. & 651. \\
700 & 8.891 & 8.896 & 0.05 & 49. & 888. \\
800 & 8.891 & 8.896 & 0.04 & 61. & 1191. \\
900 & 8.891 & 8.895 & 0.04 & 74. & 1534. \\
1000 & 8.891 & 8.895 & 0.03 & 89. & 1928. \\
\hline
\end{tabular}

Figure 3: Convergence of primal and dual SDDP for $T=12$. Time corresponds to cumulated time along iterations.

computation times needed to run both primal and dual SDDP algorithms. We observe that the upper bound Dual UB $\left[\underline{\mathcal{D}}_{0}\right]^{\star}\left(x_{0}\right)$ given by dual SDDP converges towards the primal lower bound Primal LB $\underline{V}_{0}\left(x_{0}\right)$ given by primal SDDP, with a relative gap close to $0.03 \%$ after 1,000 iterations. For this specific (with few time steps) example, the convergence of dual SDDP proves 
to be effective. As noticed at Remark 24, running dual SDDP is much more time consuming than running primal SDDP.

The outer and inner strategies are evaluated by Monte Carlo. An evaluation is performed every 50 iterations with an once for all given set of 10,000 scenarios, that is, a "large" sample. Both evaluations MC OA and MC IA converge to the optimal value. We notice that MC IA is below Dual UB, thus illustrating the result stated by Theorem 29 .

Solving the problem over a three years time horizon. We now consider the same problem, but over a three years horizon, that is, with $T=36$. The convergence of primal and dual SDDP is shown in Figure 4. Compared to Figure 3, we have materialized the confidence inter-

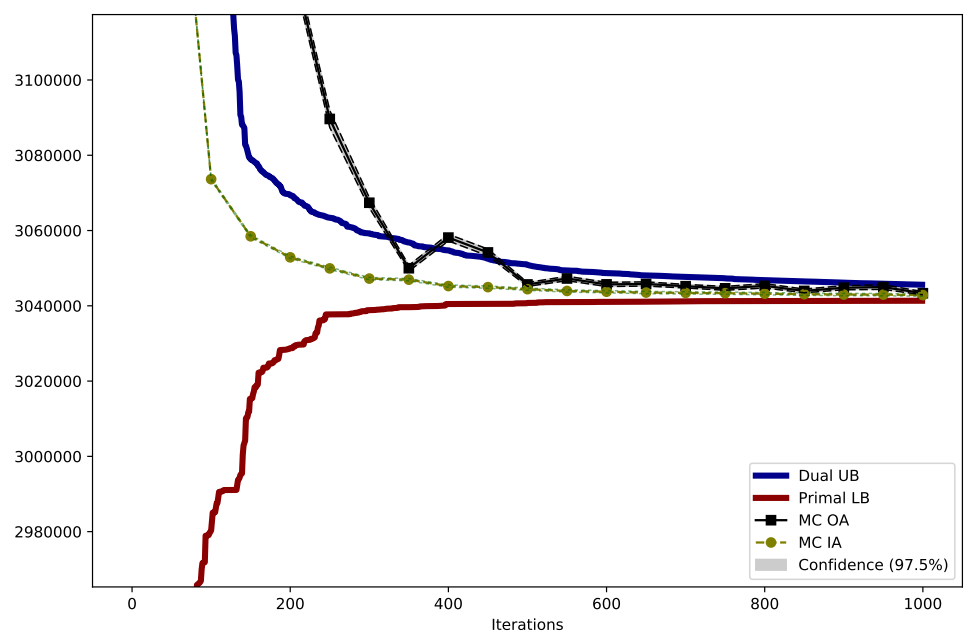

\begin{tabular}{cccccc}
\hline Iter. & Primal LB $\left(\times 10^{6}\right)$ & Dual UB $\left(\times 10^{6}\right)$ & Gap $(\%)$ & Time LB $(\mathrm{s})$ & Time UB (s) \\
\hline 50 & 2.837 & 3.917 & 38.1 & 5. & 20. \\
100 & 2.980 & 3.151 & 5.7 & 11. & 74. \\
200 & 3.029 & 3.070 & 1.4 & 27. & 267. \\
300 & 3.039 & 3.059 & 0.67 & 46. & 592. \\
400 & 3.040 & 3.055 & 0.46 & 75. & 1113. \\
500 & 3.041 & 3.051 & 0.34 & 108. & 1783. \\
600 & 3.041 & 3.049 & 0.25 & 144. & 2601. \\
700 & 3.041 & 3.048 & 0.21 & 187. & 3585. \\
800 & 3.041 & 3.047 & 0.18 & 235. & 4751. \\
900 & 3.041 & 3.046 & 0.15 & 296. & 6140. \\
1000 & 3.041 & 3.046 & 0.13 & 360. & 7545. \\
\hline
\end{tabular}

Figure 4: Convergence of primal and dual SDDP for $T=36$. Time corresponds to cumulated time along iterations.

vals (here very thin) of the inner and outer strategies Monte Carlo simulations, both estimated every 50 iterations with an once for all given set of 10,000 scenarios. A first observation is that both dual and primal SDDP exhibit a slower convergence than in the first example: after 1,000 iterations, the gap between the primal lower bound Primal LB and the dual upper bound Dual UB is equal to $0.13 \%$. This well-known behavior of SDDP arises from the increasing number of time-steps (36 instead of 12). Moreover, Dual UB is still significantly decreasing after iteration 500 , and it seems that it converges more slowly than Primal LB.

A second observation is that the dual upper bound Dual UB is better than the statistical cost 
value MC OA up to iteration 500. After the first 500 iterations, MC OA is better that Dual UB and slightly fluctuates above the primal lower bound Primal LB (the remaining gap being around $0.1 \%$ after 1,000 iterations).

Finally, on this example, the value of MC OA is greater than the value of MC IA at every iteration. Surprisingly enough, the value of MC IA exhibits a more stable behavior than the one given by MC OA. It would be interesting to be able to assess such behaviors.

\subsubsection{Using the dual upper bound in a stopping criteria}

Consider the problem over a three years time horizon. The gap between the two deterministic bounds (primal lower bound Primal LB and dual upper bound Dual UB) against the number of iterations is given in Figure 4. To complete these results, we give the evolution of the statistical upper bound MC OA UB obtained by the outer strategy in Table 1 . We aim at comparing two stopping tests.

\begin{tabular}{ccccc}
\hline Iter. & Primal LB $\left(\times 10^{6}\right)$ & Gap Dual UB $(\%)$ & MC OA UB $\left(\times 10^{6}\right)$ & Gap MC OA UB $(\%)$ \\
\hline 50 & 2.837 & 38.1 & 3.392 & 19.6 \\
100 & 2.980 & 5.7 & 3.310 & 11.1 \\
200 & 3.029 & 1.4 & 3.137 & 3.6 \\
300 & 3.039 & 0.67 & 3.069 & 1.0 \\
400 & 3.040 & 0.46 & 3.059 & 0.62 \\
500 & 3.041 & 0.34 & 3.046 & 0.18 \\
600 & 3.041 & 0.25 & 3.046 & 0.18 \\
700 & 3.041 & 0.21 & 3.046 & 0.15 \\
800 & 3.041 & 0.18 & 3.046 & 0.16 \\
900 & 3.041 & 0.16 & 3.045 & 0.14 \\
1000 & 3.041 & 0.13 & 3.044 & 0.08 \\
\hline
\end{tabular}

Table 1: Statistical upper bound for $T=36$.

Statistical stopping test: it is the stopping test proposed in Shapiro 2011 and which has been detailed at $\$ 1.2$. We choose a confidence level $\alpha=.975$, and we estimate the statistical upper bound MC OA UB every 50 iterations with a given set of 10,000 scenarios.

Dual stopping test: this stopping test is just based on the gap between the available deterministic upper and lower bounds, namely Dual UB and Primal LB.

For different accuracy levels $\varepsilon$, as described by Shapiro 2011] we compare the CPU times taken by these two tests in order to stop the SDDP algorithm. Results are given in Table 2. The given

\begin{tabular}{cccccc}
\hline & \multicolumn{2}{c}{ Dual stopping test } & & \multicolumn{2}{c}{ Statistical stopping test } \\
\cline { 6 - 6 }$(\%)$ & $n$ it. & CPU time & & $n$ it. & CPU time \\
\hline 2.0 & 156 & $183 \mathrm{~s}$ & & 250 & $618 \mathrm{~s}$ \\
1.0 & 236 & $400 \mathrm{~s}$ & & 300 & $787 \mathrm{~s}$ \\
0.5 & 388 & $1116 \mathrm{~s}$ & & 450 & $1429 \mathrm{~s}$ \\
0.1 & $>1000$ &. & & 1000 & $5519 \mathrm{~s}$ \\
\hline
\end{tabular}

Table 2: Comparing dual and statistical stopping criteria for different accuracy levels $\varepsilon$.

times correspond to the total time required to run SDDP (including both the computation of cuts and the computation of the stopping test). We notice that the dual stopping test gives better 
results than the statistical stopping test: for $\varepsilon \geq 0.45 \%$, it stops SDDP earlier and require less computation time. Compared with the statistical test, the speed-up is between 3.3 for $\varepsilon=2 \%$ and 1.3 for $\varepsilon=0.5 \%$. However, the dual stopping test is penalized by the slow convergence of dual SDDP. Indeed it cannot achieve a gap lower than $0.1 \%$, thus penalizing the performance of the dual stopping test for high accuracy levels $\varepsilon$.

As a conclusion of these numerical experiments, the deterministic dual stopping test seems to be better that the statistical stopping test, especially if restrictions on the CPU time impose to perform a limited number of SDDP iterations (less than 500 in our case). Such a situation exists in the energy field, as shown by the description of the Brazilian interconnected power system in Shapiro et al. 2012].

Remark 31. We can also use the statistical upper bound MC IA UB obtained by evaluating the inner strategy for the statistical stopping test designed by Shapiro. Indeed, in our numerical experiments, this upper bound is always lower than the one given by the outer strategy. However, this would require much longer computational time, as this approach combines the computation of the dual cuts together with a Monte-Carlo estimation.

\subsubsection{Strengths and weaknesses of dual SDDP}

Dual SDDP allows us to obtain a deterministic stopping criterion, which proves to be effective compared to the standard statistical stopping test. Furthermore, dual SDDP computes cuts that can be used to design an inner approximation strategy, which appears to be better than the outer strategy whenever primal SDDP has not exactly converged.

However, we observe that the convergence of dual SDDP is penalized by different considerations.

- It is well-known from Shapiro 2011 that the convergence of SDDP is impacted by the number of stages in the problem. This issue impacts both primal and dual SDDP.

- Furthermore, we notice that dual SDDP exhibits a slower convergence than primal SDDP. In fact, primal SDDP computes its trajectories from a fixed initial point $x_{0}$, whereas, as explained at $\$ 3.2 .2$, dual SDDP updates its initial point $\lambda_{0}$ at each iteration, with

$$
\lambda_{0}^{k} \in \underset{\left\|\lambda_{0}\right\| \leq L_{0}}{\arg \max }\left\{x_{0}^{\top} \lambda_{0}-\underline{\mathcal{D}}_{0}^{k}\left(\lambda_{0}\right)\right\} .
$$

- One iteration of dual SDDP takes longer than one iteration of primal SDDP. Indeed, dual SDDP solves bigger LP problems than primal SDDP, as it has to consider explicitly a coupling constraint (36b) between all samples. Dual SDDP would greatly benefit from a cuts selection algorithm, which would limit the number of constraints added in the problem.

\section{Conclusion}

In this paper, we have described a new method to compute exact upper bounds for SDDP, which relies on applying SDDP to the Fenchel transform of Bellman's value functions. We have shown that under classical assumptions the upper bound converges to the optimal value of the problem.

We have taken advantage of the dual value functions $\left\{\underline{\mathcal{D}}_{t}\right\}_{t \in 0, T}$ to build a sequence of inner approximations $\left\{\bar{V}_{t}\right\}_{t \in \llbracket 0, T \rrbracket}$ of the primal value functions $\left\{V_{t}\right\}_{t \in \llbracket 0, T \rrbracket}$. We proved that the policy induced by these inner approximations is converging to an optimal policy, with guaranteed performance of the expected cost associated. We tested dual SDDP with extensive numerical results 
on a stochastic production planning problem. These numerical results proved the effectiveness of dual SDDP and the underlying inner strategy. Furthermore, we showed on this particular problem that using a dual stopping test outperforms the classical statistical stopping test of SDDP, both in term of iterations number and in term of computational burden.

We plan to extend this study in several directions. First, an extension of dual SDDP to risk averse or distributionally robust problems remains to be investigated. Second, the dual SDDP algorithm used in this paper does not decompose the subproblems at each time steps uncertainty by uncertainty, so as to obtain smaller subproblems with a more tractable size. This penalizes dual SDDP towards primal SDDP, leading to greater computation times. A proper way to decompose the dual subproblems effectively is under study. Third, we want to test dual SDDP on other problem, such as the Brazilian hydro power operation planning problem presented in Shapiro et al. 2012. Finally we want to explore the interactions between primal and dual SDDP. For example, we think that the upper bounds given by dual SDDP might be effective to regularize SDDP, for instance with the method introduced by Van Ackooij et al. [2017.

\section{A Appendix}

\section{A.1 Recalls on convex analysis}

We quickly recall some results that can be found in any convex analysis book. We follow the definitions of Rockafellar [1970].

A convex extended real valued function is proper if it never takes the value $-\infty$ and is not identically equal to $+\infty$. A polyhedral subset of $\mathbb{R}^{n}$ is the finite intersection of closed half spaces, and a polyhedral function is a function whose epigraph is a polyhedral set. In particular a polyhedral function is convex l.s.c., but not necessarily proper. A non-proper polyhedral function takes value $-\infty$ on a polyhedral set, and $+\infty$ elsewhere.

Let $f: \mathbb{R}^{n} \rightarrow \overline{\mathbb{R}}$ be an extended real valued function. By definition its Fenchel conjugate $f^{\star}$ and concave conjugate $f_{\star}$ are defined as

$$
f^{\star}\left(x^{\star}\right):=\sup _{x \in \mathbb{R}^{n}}\left\langle x^{\star}, x\right\rangle-f(x) \quad f_{\star}\left(x^{\star}\right):=\inf _{x \in \mathbb{R}^{n}}\left\langle x^{\star}, x\right\rangle-f(x) .
$$

Let $f$ and $-g$ be proper polyhedral functions. Then we have the following duality result (see Rockafellar, 1970, § 31])

Proposition 32 (Fenchel-Duality). If $\operatorname{dom}(f) \cap \operatorname{dom}(-g) \neq \emptyset$, we have

$$
\inf _{x} f(x)-g(x)=\sup _{x^{\star}} g_{\star}\left(x^{\star}\right)-f^{\star}\left(x^{\star}\right) .
$$

We end these recalls by results on Lipschitz regularization. For any proper functions $f$ and $g$ of $\mathbb{R}^{n}$ we define the infimal convolution as

$$
f \square g: x \mapsto \inf _{y \in \mathbb{R}^{n}} f(y)+g(x-y) .
$$

Let $f$ be a proper function of $\mathbb{R}^{n}$, we denote $f^{L}$ its $L$-Lipschitz regularization - or Pasch Hausdorff regularization - with respect to the $L^{1}$-norm defined has

$$
f^{L}:=f \square\left(L\|\cdot\|_{1}\right) .
$$

We have the following properties (see [Bauschke et al., 2017, Corollary 12.19]) 
Proposition 33. Let $f$ be a proper function of $\mathbb{R}^{n}$, and $L$ be a positive number. Then, $f^{L}$ is the largest L-Lipschitz function that is lower than $f$. In particular we have

- $f^{L}$ is a lower approximation of $f: f^{L} \leq f$,

- $f^{L}$ is L-Lipschitz with respect to the $L_{1}$ norm,

- if $f$ is L-Lipschitz on its domain, then for all $x \in \operatorname{dom}(f), f^{L}(x)=f(x)$.

\section{A.2 Numerical settings}

We describe the problem in $\$ 5$ with dynamics and constraint matrix.

$$
A=I_{n}, \quad B=-\left(\begin{array}{lllll}
I_{n} & I_{n} & 0_{n} & 0_{n} & 0_{q n}
\end{array}\right), \quad C=\left(I_{n} 0_{n}\right),
$$

where $I_{n}$ is the identity matrix and $0_{n}$ the square null matrix with size $n$. The costs vector becomes $a_{t}=0$ and $b_{t}=\left(\begin{array}{lllll}0 & 0 & c_{t} & t_{t} & p_{t}\end{array}\right)^{\top}$. The constraints matrix write

$$
D=\left(\begin{array}{llllllllll}
0_{n} & 0_{n} & 0_{n} & 0_{n} & 0_{n} & 0_{n} & 0_{q n} & 0_{q n} & A & -A
\end{array}\right)^{\top},
$$

and

$$
E=\left(\begin{array}{ccccc}
I_{n} & 0_{n} & 0_{n} & 0_{n} & 0_{q n} \\
-I_{n} & 0_{n} & 0_{n} & 0_{n} & 0_{q n} \\
0_{n} & -I_{n} & 0_{n} & 0_{n} & 0_{q n} \\
0_{n} & 0_{n} & I_{n} & 0_{n} & 0_{q n} \\
0_{n} & 0_{n} & -I_{n} & 0_{n} & 0_{q n} \\
0_{n} & 0_{n} & 0_{n} & -I_{n} & 0_{q n} \\
0_{q n} & 0_{q n} & 0_{q n} & 0_{q n} & I_{q} \\
0_{q n} & 0_{q n} & 0_{q n} & 0_{q n} & -I_{q} \\
I_{n} & 0_{n} & I_{n} & I_{n} & R \\
& B & & & \\
& -B & & &
\end{array}\right),
$$

and

$$
g_{t+1}=\left(\begin{array}{lllllllllllll}
\bar{q} & 0 & 0 & \bar{g} & 0 & 0 & \bar{f} & \underline{f} & \boldsymbol{d}_{t+1} & \boldsymbol{d}_{t+1} & \left(\bar{v}-\boldsymbol{a}_{t+1}\right) & \boldsymbol{a}_{t+1}
\end{array}\right)^{\top}
$$




\section{A.3 Exhaustive primal-dual SDDP algorithm}

Data: Lipschitz bounds $N L_{t \in \in[0, T \rrbracket}$

for $k=0,1, \ldots$ do

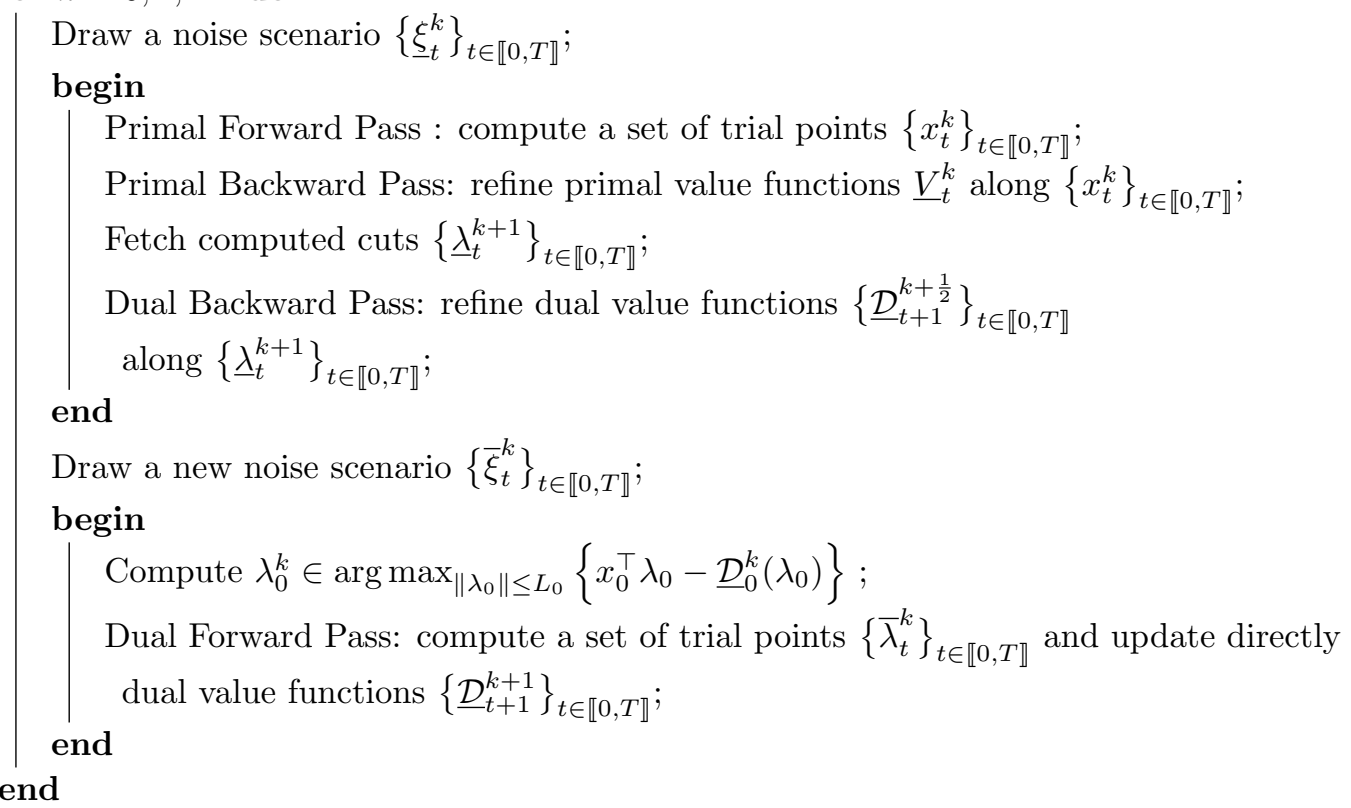

Algorithm 4: Primal-Dual SDDP algorithm

\section{A.4 Compatibility of the primal and dual Bellman operators}

We consider Problem (1) and its associated recursive Bellman equation:

$$
\begin{aligned}
& V_{T}(x)=K(x), \\
& V_{t}(x)=\inf _{\boldsymbol{U}, \boldsymbol{Y}} \mathbb{E}\left[a_{t}^{\top} x+b_{t+1}^{\top} \boldsymbol{U}+V_{t+1}(\boldsymbol{Y})\right], \\
& \text { s.t. } \quad \boldsymbol{Y}=A_{t} x+B_{t+1} \boldsymbol{U}+C_{t+1} \boldsymbol{\xi}_{t+1}, \\
& \boldsymbol{U} \in\left[\underline{u}_{t+1}, \bar{u}_{t+1}\right], \\
& \boldsymbol{Y} \in\left[\underline{x}_{t+1}, \bar{x}_{t+1}\right] \\
& D_{t} x+E_{t+1} \boldsymbol{U}+G_{t+1} \boldsymbol{\xi}_{t+1} \leq 0 .
\end{aligned}
$$

Ignoring the constant term $a_{t}^{\top} x$, this equation rewrites as a linear Bellman operator

$$
\begin{array}{rl}
\mathcal{B}_{t}\left(V_{t+1}\right)(x)=\inf _{\boldsymbol{U}, \boldsymbol{Y}} & \mathbb{E}\left[b_{t+1}^{\top} \boldsymbol{U}+V_{t+1}(\boldsymbol{Y})\right], \\
\text { s.t. } & T x+\mathcal{W}_{u}(\boldsymbol{U})+\mathcal{W}_{y}(\boldsymbol{Y}) \leq \boldsymbol{H},
\end{array}
$$


with the notation:

$$
T=\left[\begin{array}{c}
A_{t} \\
-A_{t} \\
0 \\
0 \\
0 \\
0 \\
D_{t}
\end{array}\right] \quad, \quad \mathcal{W}_{u}=\left[\begin{array}{c}
B_{t+1} \\
-B_{t+1} \\
I \\
-I \\
0 \\
0 \\
E_{t+1}
\end{array}\right] \quad, \quad \mathcal{W}_{y}=\left[\begin{array}{c}
-I \\
I \\
0 \\
0 \\
I \\
-I \\
0
\end{array}\right] \quad, \quad \boldsymbol{H}=\left[\begin{array}{c}
-C_{t+1} \boldsymbol{\xi}_{t+1} \\
C_{t+1} \boldsymbol{\xi}_{t+1} \\
\bar{u}_{t+1} \\
-\underline{u}_{t+1} \\
\bar{x}_{t+1} \\
-\underline{x}_{t+1} \\
-G_{t+1} \boldsymbol{\xi}_{t+1}
\end{array}\right]
$$

Denoting by $\boldsymbol{\mu}=\left(\boldsymbol{\mu}_{1}, \ldots, \boldsymbol{\mu}_{7}\right)$ the multiplier associated to the constraint of this problem, the Fenchel conjugate of $\mathcal{B}_{t}\left(V_{t+1}\right)$

$$
\begin{aligned}
\mathcal{B}_{t}^{\dagger}\left(V_{t+1}^{\star}\right)(\lambda)=\inf _{\boldsymbol{\mu}, \boldsymbol{\nu}} & \mathbb{E}\left[-\boldsymbol{\mu}^{\top} \boldsymbol{H}+V_{t+1}^{\star}(\boldsymbol{\nu})\right], \\
\text { s.t. } & T^{\top} \mathbb{E}[\boldsymbol{\mu}]+\lambda=0, \\
& \mathcal{W}_{u}^{\dagger}(\boldsymbol{\mu})=b_{t+1}, \\
& \mathcal{W}_{y}^{\dagger}(\boldsymbol{\mu})=\boldsymbol{\nu}, \\
& \boldsymbol{\mu} \leq 0,
\end{aligned}
$$

writes:

$$
\begin{aligned}
\mathcal{B}_{t}^{\ddagger}\left(V_{t+1}^{\star}\right)(\lambda)=\inf _{\boldsymbol{\mu}, \boldsymbol{\nu}} & \mathbb{E}\left[\left(\boldsymbol{\mu}_{1}-\boldsymbol{\mu}_{2}\right)^{\top} C_{t+1} \boldsymbol{\xi}_{t+1}-\boldsymbol{\mu}_{3}^{\top} \bar{u}_{t+1}+\boldsymbol{\mu}_{4}^{\top} \underline{u}_{t+1}-\boldsymbol{\mu}_{5}^{\top} \bar{x}_{t+1}\right. \\
& \left.+\boldsymbol{\mu}_{6}^{\top} \underline{x}_{t+1}+\boldsymbol{\mu}_{7}^{\top} G_{t+1} \boldsymbol{\xi}_{t+1}+V_{t+1}^{\star}(\boldsymbol{\nu})\right], \\
\text { s.t. } \quad & A_{t}^{\top} \mathbb{E}\left[\boldsymbol{\mu}_{1}-\boldsymbol{\mu}_{2}\right]+D_{t}^{\top} \mathbb{E}\left[\boldsymbol{\mu}_{7}\right]=-\lambda, \\
& B_{t+1}^{\top}\left(\boldsymbol{\mu}_{1}-\boldsymbol{\mu}_{2}\right)+\left(\boldsymbol{\mu}_{3}-\boldsymbol{\mu}_{4}\right)+E_{t+1}^{\top} \boldsymbol{\mu}_{7}=b_{t+1}, \\
& -\left(\boldsymbol{\mu}_{1}-\boldsymbol{\mu}_{2}\right)+\left(\boldsymbol{\mu}_{5}-\boldsymbol{\mu}_{6}\right)=\boldsymbol{\nu}, \\
& \boldsymbol{\mu}_{1} \leq 0, \quad \boldsymbol{\mu}_{2} \leq 0, \quad \boldsymbol{\mu}_{3} \leq 0, \quad \boldsymbol{\mu}_{4} \leq 0, \quad \boldsymbol{\mu}_{5} \leq 0, \quad \boldsymbol{\mu}_{6} \leq 0, \quad \boldsymbol{\mu}_{7} \leq 0 .
\end{aligned}
$$

We make the following assumption.

\section{Assumption 4.}

$$
\forall \lambda \in \mathbb{R}^{n}, \exists \mu_{a} \in \mathbb{R}^{n}, \exists \mu_{b} \in \mathbb{R}^{p}, \mu_{b} \leq 0 \text { such that } A_{t}^{\top} \mu_{a}+D_{t}^{\top} \mu_{b}+\lambda=0 .
$$

Remark 34. Such an assumption is for example fulfilled if $A_{t}$ is a (square) full rank matrix. $\diamond$

Under Assumption 4 and with any arbitrary non positive random variables $\boldsymbol{\mu}_{5}$ and $\boldsymbol{\mu}_{6}$, the pair of random vectors $(\boldsymbol{\mu}, \boldsymbol{\nu})$ defined by

- $\boldsymbol{\mu}=\left(\left(\mu_{a}\right)^{-},-\left(\mu_{a}\right)^{+},\left(b_{t+1}-B_{t+1}^{\top} \mu_{a}-E_{t+1}^{\top} \mu_{b}\right)^{-},-\left(b_{t+1}-B_{t+1}^{\top} \mu_{a}-E_{t+1}^{\top} \mu_{b}\right)^{+}, \boldsymbol{\mu}_{5}, \boldsymbol{\mu}_{6}, \mu_{b}\right)$,

- $\boldsymbol{\nu}=-\mu_{a}+\left(\boldsymbol{\mu}_{5}-\boldsymbol{\mu}_{6}\right)$,

satisfies the constraints of the optimization problem associated to the computation of $\mathcal{B}_{t}^{\ddagger}\left(V_{t+1}^{\star}\right)(\lambda)$. Such a pair $(\boldsymbol{\mu}, \boldsymbol{\nu})$ exists for all possible values of $\lambda$. Moreover, $\boldsymbol{\nu}$ linearly depends on the difference $\boldsymbol{\mu}_{5}-\boldsymbol{\mu}_{6}, \boldsymbol{\mu}_{5}$ and $\boldsymbol{\mu}_{6}$ being any arbitrary negative random variables, so that $\boldsymbol{\nu}$ can take any possible value in $\mathbb{R}^{n}$. 
We thus deduce that the domain of the dual constraint set valued mapping $\mathcal{G}_{t}^{\ddagger}$, with

$$
\mathcal{G}_{t}^{\ddagger}(\lambda)=\left\{(\boldsymbol{\mu}, \boldsymbol{\nu}) \in \mathbb{R}^{n_{x}+n_{c}} \mid T^{\top} \mathbb{E}[\boldsymbol{\mu}]+\lambda=0, \mathcal{W}_{u}^{\dagger}(\boldsymbol{\mu})=\boldsymbol{C}, \mathcal{W}_{y}^{\dagger}(\boldsymbol{\mu})=\boldsymbol{\nu}, \boldsymbol{\mu} \leq 0\right\},
$$

is equal to the whole space $\mathbb{R}^{n}$, so that the sequence of dual linear Bellman operators $\left(\mathcal{B}_{t}^{\ddagger}\right)_{t \in \llbracket 0, T-1 \rrbracket}$ is compatible.

\section{References}

Regan Baucke, Anthony Downward, and Golbon Zakeri. A deterministic algorithm for solving multistage stochastic programming problems. Optimization Online, 2017.

Heinz H Bauschke, Patrick L Combettes, et al. Convex analysis and monotone operator theory in Hilbert spaces, volume 2011. Springer, 2017.

Richard Bellman. Dynamic Programming. Princeton University Press, New Jersey, 1957.

Dimitri P Bertsekas. Dynamic programming and optimal control, volume 1 \& 2. Athena Scientific Belmont, MA, 3rd edition, 2005.

Jonathan Borwein and Adrian S Lewis. Convex analysis and nonlinear optimization: theory and examples. Springer, 2010.

Iain Dunning, Joey Huchette, and Miles Lubin. JuMP: A modeling language for mathematical optimization. SIAM Review, 59(2):295-320, 2017.

Pierre Girardeau, Vincent Leclere, and Andrew B Philpott. On the convergence of decomposition methods for multistage stochastic convex programs. Mathematics of Operations Research, 40 (1):130-145, 2014.

Vincent Guigues. Convergence analysis of sampling-based decomposition methods for risk-averse multistage stochastic convex programs. SIAM Journal on Optimization, 26(4):2468-2494, 2016.

Vincent Guigues. Dual dynamic programing with cut selection: Convergence proof and numerical experiments. European Journal of Operational Research, 258(1):47-57, 2017.

Tito Homem-de Mello, Vitor L De Matos, and Erlon C Finardi. Sampling strategies and stopping criteria for stochastic dual dynamic programming: a case study in long-term hydrothermal scheduling. Energy Systems, 2(1):1-31, 2011.

Philippe Mahey, Jonas Koko, and Arnaud Lenoir. Decomposition methods for a spatial model for long-term energy pricing problem. Mathematical Methods of Operations Research, 85(1): 137-153, 2017.

Mario VF Pereira and Leontina MVG Pinto. Multi-stage stochastic optimization applied to energy planning. Mathematical programming, 52(1-3):359-375, 1991.

Andrew Philpott and Ziming Guan. On the convergence of stochastic dual dynamic programming and related methods. Operations Research Letters, 36(4):450-455, 2008.

Andrew Philpott, Vitor de Matos, and Erlon Finardi. On solving multistage stochastic programs with coherent risk measures. Operations Research, 61(4):957-970, 2013. 
R Tyrrell Rockafellar. Convex analysis. Princeton University Press, 1970.

R Tyrrell Rockafellar and Roger JB Wets. Stochastic convex programming: relatively complete recourse and induced feasibility. SIAM Journal on Control and Optimization, 14(3):574-589, 1976.

Alexander Shapiro. Analysis of stochastic dual dynamic programming method. European Journal of Operational Research, 209(1):63-72, 2011.

Alexander Shapiro, Darinka Dentcheva, and Andrzej Ruszczyński. Lectures on stochastic programming: modeling and theory. SIAM, 2009.

Alexander Shapiro, Wajdi Tekaya, Joari P da Costa, and Murilo P Soares. Final report for technical cooperation between georgia institute of technology and ons-operador nacional do sistema elétrico. Georgia Tech ISyE Report, 2012.

Wim Van Ackooij, Welington de Oliveira, and Yongjia Song. On regularization with normal solutions in decomposition methods for multistage stochastic programming. Optimization Online, 2017. 